\title{
Radically elementary analysis of an interacting particle system at an unstable equilibrium
}

\author{
HEINZ WEISSHAUPT $^{1}$
}

\begin{abstract}
We investigate an interacting particle system consisting of two types of particles located at a finite point-lattice. The particles randomly change their type and neighboring particles randomly interchange positions. The system seems to remain at equilibrium for a substantial amount of time until it suddenly, at a critical time $T$, leaves equilibrium along what seems to be a deterministic trajectory. The analysis reveals, however, that the trajectories are determined randomly, but only by the systems behavior at very early times, much prior to $T$. In the nonstandard model used, the system randomly 'chooses' the trajectory in an infinitesimal interval $[0, \varepsilon], \varepsilon \approx 0$, but this choice only becomes visible in the interval $[T-\varepsilon, T]$. The underlying reason for this behavior is revealed by a decomposition of the systems trajectories with respect to an eigenbasis $\left(g_{k}\right)_{k \in \mathcal{K}}$ of the discrete Laplace operator $\triangle$. It shows that after an initial random period the system's dynamics behaves, coordinate-wise, like $t \mapsto e^{\left(\lambda+\mu_{k}\right)(t-T)} v_{k}(\omega)$, where $\lambda$ is unlimited ('infinitely large'), $\mu_{k} g_{k}=\triangle g_{k}$ and $v_{k}(\omega)$ denotes a random quantity. The hyperfinite result obtained is translated into a standard limit theorem.
\end{abstract}

2000 Mathematics Subject Classification 26E35 (primary); 82C22, 60J60, 60F05, $82 \mathrm{C} 20,60 \mathrm{~J} 10$ (secondary)

Keywords: interacting particle systems, limit laws, random induced coherence, spectral decomposition, reaction-diffusion processes, hyperfinite approximation, infinitesimals, nonstandard analysis, zero-range processes

\section{Introduction}

Interacting particle systems have been a prospering field of mathematical studies in a standard setting (Griffeath [11] and Liggett [15]) as well as a nonstandard one (Helms and Loeb [12], and Albeverio, Fenstad, Høegh-Krohn and Lindstrøm [1, Chapter 7]), the most prominent being the Ising model.

\footnotetext{
${ }^{1}$ Research has been supported by the BMBF, Germany, through FRISYS (Freiburg Initiative for Systems biology), Kennzeichen 0313921.
} 
The model under consideration is presented within a nonstandard setting. It shares with the Ising model the property of being a Markovian lattice model and that there exist two states for each particle, or equivalently that there are two particle types, or particles and holes. It differs, however, in that a large number of particles occupies one position at a time. In this regard, it possesses similarities with discrete-time zero-range processes (in the sense of Evans and Hanney [10]) or reaction diffusion processes (in the sense of Chen [7, Section 13.2]).

The system's dynamics is at first defined only if particles of both types are present at any position.

We investigate the evolution starting in the unique unstable equilibrium of a corresponding deterministic system (briefly discussed in Remark 5.5). We are only interested in the way the system leaves this equilibrium. This can equally well be investigated within any extension of the original system. Thus we extend the system's dynamics in a mathematically appropriate way to arbitrary (negative, real valued) quantities of particles. For the sake of simplicity, we describe the extended dynamics by the deviation of the pointwise particle concentration from the equilibrium.

The system's evolution can be divided into three periods. The first and the third period are very short compared to the second one. During the first and second period the system stays infinitesimally close to the unstable equilibrium, and during the third period it drives with high velocity away from this initial state.

In the first period the system's evolution is particularly governed by stochasticity. In the second and third one each path of the system stays infinitesimally close to a deterministic trajectory. $^{2}$ Thus the system's behavior in periods two and three is approximately described by a probability distribution on a family of deterministic trajectories. The effect of stochasticity in periods two and three, therefore, originates approximately from a random choice of a deterministic trajectory made during period one, while the additional influence of randomness during periods two and three is rather negligible.

To obtain an intuition for the system's behavior, suppose that we are unable to recognize infinitesimal differences. Then the system seems to stay in equilibrium during periods one and two. In period three we observe that the system drives away from the unstable equilibrium along a randomly chosen, but deterministic trajectory. We know, however, that the system has already come to the random decision for this particular trajectory during period one.

\footnotetext{
${ }^{2} \mathrm{We}$ use the terms trajectory and path in the sense of time-indexed families of states/configurations, i.e., trajectories and path are functions from time into the state space of our dynamical system.
} 
The deterministic trajectories associated with the system are solutions of a linear system of first order infinitesimal difference equations $y_{t+\delta t}=L y_{t}$, where the linear transformation $L$ is diagonalizable with respect to an eigenbasis of the discrete Laplace operator. Stochastically the system shows a Gaussian behavior: Projections of the system's random-state onto orthogonal eigenvectors of the Laplacian are approximately independent, approximately normally distributed random variables. The variances of these variables increase geometrically with time. The velocity of the increase depends on the corresponding eigenvalues of the Laplacian. This leads to a preference of low frequencies and represents a certain degree of coherence induced by stochasticity, although the term 'stochastic coherence' seems usually to be associated only with nonlinear systems (e.g. Sagues, Sancho and Garcia-Ojalvo [23]).

We are interested in the system's behavior for large numbers of particles. This is within standard mathematics expressed by limit theorems. Largeness can however be directly expressed within a nonstandard framework. In such a setting hyperfinite collections are large compared to standard finite ones. It is further possible to obtain from results concerning the hyperfinite situation corresponding limit results in standard mathematical terms. In this way Lindeberg type limit theorems have been proved in Weisshaupt [28]. Following this idea we characterize the system's dynamics for hyperfinite particle-collections first (Theorem 6.5), and apply afterward transfer and the permanence principle to obtain a corresponding standard limit result (Theorem 7.9). The article follows Nelson's axiomatic approach IST [18] to nonstandard analysis. It is radically elementary in the sense that it is based on (hyper)-finite probability spaces and the IST-axioms of idealization and transfer, while the IST-axiom of standardization is not used in the whole article. Only in the formulation of Corollary 7.11 do we make use of uncountable probability spaces, since the standard limit object involved can not be defined on a finite probability space. For this reason we also included appendix B that connects our internal concepts to standard measure theoretic ones. Note however, that appendix B is still radically elementary in the sense that it only uses idealization and transfer to establish this connection.

\section{Organization of the Article}

In Section 3 we describe the basic dynamics of the interacting particle system and indicate how this dynamics relates to the extended dynamics defined in Section 5. We further outline the main result and discuss the outline in some detail. We briefly indicate how our simple interacting particle systems may relate to more complex systems in chemical reaction kinetics. Finally we discuss the main proof-steps. 
Section 4 introduces some fundamental notions and results in nonstandard analysis like infinitesimals, uniform S-continuity, near intervals and the symbol $\oslash$. We further introduce the discrete Laplace operator and its eigenbasis, which becomes in Section 5 the fundamental tool for the investigation of the extended dynamics. Finally the concepts of conditional probability, partially defined random variable, stochastic process and approximately normally distributed variable are introduced.

In Section 5 we introduce the extended model in a mathematically self contained way not relying on Section 3, however without the motivation and explanation already given before. The main purpose of Section 5 is to obtain a description of the extended dynamics in coordinates with respect to the eigenbasis of the discrete Laplacian introduced in Section 4.

In Section 6 we prove the main results of this article in their internal form (Theorem 6.5 and Theorem 6.9). Both theorems describe the coordinate-wise deviation (with respect to the eigenbasis of the discrete Laplace operator) of the system from deterministic trajectories. While Theorem 6.5 deals with the case of a small (standard finite) number of available particle-positions, the Theorem 6.9 is concerned with the hyperfinite case. The proof of Theorem 6.5 is based on Theorem A.7, the description of the system's dynamics obtained in Section 5 and the Doob inequality (stated as Proposition C.3). Theorem 6.9 is a consequence of Theorem 6.5 and the axion of idealization.

Section 7 finally turns Theorem 6.5 into the standard limit Theorem 7.9. For this purpose the mathematical objects in the preceding sections have to be replaced by standard sequences. The relations fulfilled by the nonstandard elements of these sequences coincide with the relations fulfilled by the objects of the preceding sections. To obtain standard limit theorems we translate these relations into assertions concerning the limits of these sequences. It turns out that this is possible without the use of the standardization-axiom.

Appendix A is concerned with the internal central limit theorems A.5 and A.7. Under the hypotheses of these theorems the concatenation of a group homomorphism into the real numbers with the final state of certain Markov chains (on abelian groups) is approximately normally distributed. The proof of Theorem A.5 exploits the relationship between infinitesimal diffusion processes and the diffusion equation in analogy with Weisshaupt [28], while Theorem A.7 is just a modification of Theorem A.5 obtained by a time transform. We regard these theorems—as well as their proofs—as interesting in their own right.

Appendix B relates our external concept of an 'approximately $N(0, i d)$ distributed random variable' (Definition 4.22) via the Cramer-Wold device to the standard concept 
of 'convergence toward a $N(0, i d)$ distributed random variable', while Appendix C collects miscellaneous results.

The article is largely self-contained. It only makes use of some very elementary results from nonstandard analysis (Remark 4.3), elementary facts concerning discrete Fourier analysis and the discrete Laplacian (also collected in Section 4), the well-known Doob inequality (displayed for the readers convenience at the end of Appendix C) and a consequence (Proposition B.4) of the Cramér-Wold device. We do not make use of other auxiliary results. We especially state and prove in Appendix A a central limit theorem along the lines of [28] that is fundamental for the proof of our main results. Note however that it would have been possible to apply the martingale central limit theorem (Bhattacharya and Majumdar [4, Section 5.4, Proposition 4.1]) to prove our main results instead.

\section{Description of the basic dynamics}

The particle systems under consideration consist of a constant finite number $N$ of particles described by their position and their type. At a given time-point $t$ a particle possesses a position $x$ in the finite point lattice $\mathcal{H}:=h \mathbb{Z} / \mathbb{Z}$ with $1 / h \in \mathbb{N}$ and is either of type $A$ or of type $B$.

We suppose for arbitrary $x \in \mathcal{H}$ that the number of particles located at $x$ is independent of time and equals $h N \in \mathbb{N}$. We further assume that particles of the same type are only distinguished by their position, but are otherwise indistinguishable. Thus at any time $t$ the system is completely described by the spatial distribution of type- $A$ or type- $B$ particles.

Using a nonstandard framework it is convenient to model time by near intervals $[0 \ldots T]$, i.e., hyperfinite — and thus discrete—subsets of $[0, T]$ introduced in Definition 4.4, and to denote small time steps corresponding to the spacings of points in near intervals by $\delta t$.

In a small piece of time $\delta t$, one particle may change its type and two neighboring particles may interchange their position. Which particles interchange and if there is any interchange at all is a uniformly distributed pure random event independent of the particle-configuration. The probability that a particular particle at position $\mathrm{x}$ changes its type also depends on the configuration, at position $\mathrm{x}$. The influence of randomness on the system is expressed by random elements $\omega$ in some hyperfinite space $\Omega$. 
We describe the random evolution of our particle system by consecutive reaction and diffusion steps. We suppose that the reaction steps take place in the time intervals $(t, t+\delta t / 2]$ while the diffusion steps follow in $(t+\delta t / 2, t+\delta t]$, with the time-points $t$ being elements of the discrete set $[0 \ldots T]$. Instead of $t+\delta t / 2$ we write $t^{+}$. In a reaction step a particle may change its type, while in a diffusion step two particles may interchange. It is sufficient to describe the interchange of particles of different types in the diffusion step, since we are unable to observe the interchange of particles of the same type.

Let $N_{A, t}(\omega) \in\{0, \ldots, h N\}^{\mathcal{H}}$ denote the number of type-A particles at time $t$ under the random influence $\omega$ at different positions $x \in \mathcal{H}$ before the reaction step. Let further $N_{A, t^{+}}(\omega) \in\{0, \ldots, h N\}^{\mathcal{H}}$ denote the number of type-A particles at time $t^{+}$under the random influence $\omega$ at different positions $x \in \mathcal{H}$ before the diffusion step. The evolution of the system can be described by the functions $t \mapsto N_{A, t}$ and $t \mapsto N_{A, t^{+}}$, with $t \in[0 \ldots T]$. We note that the evolution of the system can equivalently be described by the number of type-B particles given by $h N-N_{A, t}$ and $h N-N_{A, t^{+}}$. We further let $\epsilon_{j, k}=1$ if $j=k$ and $\epsilon_{j, k}=0$ for $j \neq k$ and define functions $e_{x}: \mathcal{H} \rightarrow\{0,1\}$ by $e_{x}(y):=\epsilon_{x, y}$ and $\mathbb{I}_{\mathcal{K}}: \mathbb{Z} \rightarrow\{0,1\}$ by $\mathbb{I}_{\mathcal{K}}(x):=\sup _{k \in \mathcal{K}} \epsilon_{k, x}$.

Considering particles of type $A$ only and regarding particles of type $B$ as holes (free space that may be occupied by particles of type $A$ ), our dynamical system is described by hopping of particles to neighboring positions ${ }^{3}$ (instead of an interchange of particles) and the overall particle number is not conserved any more. It shares these properties with discrete-time zero-range processes with non-conservation of particle numbers in the sense of [10] or reaction-diffusion processes in the sense of [7, Section 13.2]. Fluid limits of reaction-diffusion processes have been considered in [7, Chapter 16] and Boldrighini, De Masi and Pellegrinotti [6]. Condensation phenomena for zero-range process with non-conservation of particle numbers have been investigated in Angel, Evans, Levine and Mukamel [3]. The dynamics considered in all these instances differ from ours in at least three points: Unstable equilibria (similar to ours) are not investigated (and thus obtained results are entirely different), hopping rates of particles do not depend on the occupation number at a neighboring site and reaction-rates are not 'infinitely' large compared to diffusion-rates.

We describe the reaction and the diffusion steps in more detail:

\footnotetext{
${ }^{3}$ Note that in such a description the hopping rates of particles in our dynamical system do depend on particle concentration at neighboring sites.
} 


\section{Reaction step:}

For $N_{A, t}(\omega)=n_{A, t} \in\{1, \ldots, h N-1\}^{\mathcal{H}}$ we let

$$
\begin{aligned}
N_{A, t^{+}}(\omega) & :=N_{A, t}(\omega)+Q_{A, t}(\omega) \text { with } \\
Q_{A, t}(\omega) & \in\{0\} \cup \bigcup_{x \in \mathcal{H}}\left\{-e_{x}, e_{x}\right\}
\end{aligned}
$$

i.e., the number of type-A particles remains unchanged or changes at exactly one position by \pm 1 . This formalizes the fact that in one reaction step at most one particle in the system reacts, i.e., changes its type.

The conditional probabilities (Definition 4.15) for these reactions/changes are given by:

$$
\begin{aligned}
\mathbb{P}\left(Q_{A, t}=e_{x} \mid N_{A, t}=n_{A, t}\right) & :=\delta t \frac{\lambda}{2} n_{A, t}(x), \\
\mathbb{P}\left(Q_{A, t}=-e_{x} \mid N_{A, t}=n_{A, t}\right) & :=\delta t \frac{\lambda}{2}\left(h N-n_{A, t}(x)\right)
\end{aligned}
$$

By equation (3) the probability that one of the type-B particles located at position $x$ reacts to a type-A particle is proportional to the number of type A particles located at $x$, while by equation (4) the same statement holds true with the particle-types interchanged.

Diffusion step:

For $N_{A, t^{+}}(\omega)=n_{A, t^{+}} \in\{1, \ldots, h N-1\}^{\mathcal{H}}$ we let

$$
\begin{aligned}
N_{A, t+\delta t}(\omega) & :=N_{A, t^{+}}(\omega)+Q_{A, t^{+}}(\omega) \text { with } \\
Q_{A, t^{+}}(\omega) & \in\{0\} \cup \bigcup_{x \in \mathcal{H}}\left\{-e_{x}+e_{x-h},-e_{x}+e_{x+h}\right\},
\end{aligned}
$$

i.e., the system remains unchanged or the number of type-A particles at some position $x$ decreases by 1 while the number of type-A particles at position $x-h$ or $x+h$ increases by 1 . This formalizes the interchange of a type-A particle at some position $x$ with a type-B particle at a neighboring position.

The probabilities for this interchange of particles are given by:

$$
\mathbb{P}\left(Q_{A, t^{+}}=-e_{x}+e_{x \pm h} \mid N_{A, t^{+}}=n_{A, t^{+}}\right):=\frac{\delta t}{h^{2}} n_{A, t^{+}}(x)\left(1-\frac{n_{A, t^{+}}(x \pm h)}{h N}\right)
$$

i.e., the probability that one of the type A particles located at position $x$ interchanges with a type B particle at a neighboring position is proportional to the number of type A particles located at $x$ and proportional to the concentration of type $B$ particles located at the neighboring position. 
We are interested in the system's dynamics when the reaction rate $\lambda$ in equations (3) and (4) is large compared to 1, i.e., we are interested in situations when reactions occur much more frequently than interchanges of particles.

Extended dynamics

Note that the reaction and diffusion steps have till now only been defined if

$$
N_{A, t}(\omega), N_{A, t^{+}}(\omega) \in\{1, \ldots, h N-1\}^{\mathcal{H}} .
$$

An extension of this system's dynamics is introduced in Section 5. It is based on random variables $\Xi_{t}$ and $\Xi_{t^{+}}$that fulfill $\Xi_{t}(\omega)=\frac{N_{A, t}(\omega)}{h N}-\frac{1}{2}$ and $\Xi_{t^{+}}(\omega)=\frac{N_{A, t^{+}}(\omega)}{h N}-\frac{1}{2}$ as long as $N_{A, t}(\omega), N_{A, t^{+}}(\omega)$ are defined. Note that the random variables $\Xi_{t}$ and $\Xi_{t^{+}}$model the deviations of generalized concentrations of type-A particles from $1 / 2$. They may take on arbitrary values in $\mathbb{R}^{\mathcal{H}}$. Some of these values do not correspond to actual particle numbers and can not be interpreted as actual particle concentrations. However, up to the random time

$$
\tau_{\omega}:=\max \left\{t \in[0 \ldots T] \mid \min _{x \in \mathcal{H}}\left[N_{A, t}(\omega)\right](x) \geq 2 \text { and } \max _{x \in \mathcal{H}}\left[N_{A, t}(\omega)\right](x) \leq h N-2\right\}
$$

the original and the extended system are indistinguishable. The fact that the effects we are interested in are caused while $t \leq \tau_{\omega}$ ensures that the extended dynamics captures the behavior of the particle system.

Outline of the main result

We now outline the main result of the paper. A stronger coordinate-wise version is provided by Theorem 6.5. The following outline as well as Theorem 6.5 are formulated within a nonstandard setting. A corresponding formulation as a limit theorem is provided by Theorem 7.9.

3.1 Theorem Suppose that the particle number $N$ is hyperfinite ${ }^{4}$ and that $h$ and thus $\mathcal{H}=h \mathbb{Z} / \mathbb{Z}$ is standard. Let the reaction rate $\lambda$ be such that

$$
e^{2 \lambda T}=4 h N \text { for some limited } T \in(0, \infty) .
$$

Let the length of the time steps $\delta t$ be a constant $\delta$ independent of $t$ and sufficiently small. Suppose that the initial state of the system is given by $\Xi_{0}=0$ and that the evolution of the dynamical system is governed by Definition 5.3. Then there exists an approximately $N(0, i d)$ distributed random variable $\Gamma_{T}: \Omega \rightarrow \mathbb{R}^{\mathcal{H}}$ and a jointly diagonalizable family $\left(\Phi_{-t}\right)_{t \in[0 \ldots T]}$ of linear mappings $\Phi_{-t}: \mathbb{R}^{\mathcal{H}} \rightarrow \mathbb{R}^{\mathcal{H}}$ (Definition

\footnotetext{
${ }^{4}$ For a definition of the terms 'hyperfinite', 'limited' and 'appreciable' see Notation 4.1.
} 
6.2) such that for any unlimited $\nu \in(0, \infty)$ with $\nu / \lambda \in(0 \ldots T)$ infinitesimal, such that $T-\nu / \lambda \in(\nu / \lambda \ldots T)$ and any standard $\varepsilon>0$

$$
\mathbb{P}\left(\Gamma_{T} \neq 0 \wedge \max _{t \in[\nu / \lambda \ldots T]} \frac{\left\|\Xi_{t}(\omega)-\left[\Phi_{-t} \circ \Gamma_{T}\right](\omega)\right\|_{2}^{2}}{\left\|\Phi_{-t} \circ \Gamma_{T}(\omega)\right\|_{2}^{2}} \geq e^{-\nu(1-\varepsilon)}\right) \leq \varepsilon .
$$

Further for any standard $\varepsilon>0$

$$
\mathbb{P}\left(\max _{t \in[0 \ldots T-\nu / \lambda]}\left\|\Xi_{t}\right\|_{2}^{2} \geq e^{-\nu} h^{-1}\right) \leq e^{-\nu}\left(h^{-1}+\varepsilon\right) .
$$

3.2 Remark Theorem 3.1 is a consequence of Theorem 6.5 and Corollary 6.7. A proof is given in Section 6.

\section{Discussion}

Equation (8) relates the reaction rate $\lambda$, the overall particle-number $N$, the number of available positions $1 / h$ and the approximate time $T$ it takes till an effect becomes visible. If $\ln (1 / h)$ is small compared to $\ln (N)$ (as it is under the hypothesis that $h$ is standard and $N$ is hyperfinite), then $T$ equals approximately $\ln (N) / 2 \lambda$ and the influence of $h$ on $T$ is negligible. For times smaller than $T-\nu / \lambda$ the system stays by (10) infinitesimally close to 0 , while for times larger than $\nu / \lambda$ it shows by (9) already an approximately deterministic behavior. The system 'approximately decides' in the first time period $[0 \ldots \nu / \lambda)$ for some deterministic trajectory $\left(y_{t}(\omega)\right)_{t \in[\nu / \lambda \ldots T]}:=$ $\left(\left[\Phi_{-t} \circ \Gamma_{T}\right](\omega)\right)_{t \in[\nu / \lambda \ldots T]}$. It will follow $\left(y_{t}(\omega)\right)_{t \in[\nu / \lambda \ldots T]}$ during the second time period $[\nu / \lambda \ldots T-\nu / \lambda)$, when it stays infinitesimally close to 0 , and during the third time period $[T-\nu / \lambda \ldots T]$, when it takes on appreciable values.

By Remark 6.6 the deterministic trajectory $\left(y_{t}(\omega)\right)_{t \in[\nu / \lambda \ldots T]}$ fulfills $y_{t+\delta t}=L y_{t}$ with $L$ the linear transformation given by $L g_{k}=\left(1+\mu_{k} \delta t\right)(1+\lambda \delta t) g_{k}$, where $\left(g_{k}\right)_{k \in \mathcal{K}}$ denotes an eigenbasis of the discrete Laplace operator and $\mu_{k}$ denotes the eigenvalue corresponding to $g_{k}$.

Our investigations are partially motivated by the following simple chemical reaction system:

$$
2 A+B \rightarrow 3 A \text { and } 2 B+A \rightarrow 3 B,
$$

Let $[A]$ and $[B]$ denote the concentrations of the chemical species $A$ and $B$. Suppose that $[A]+[B]=1$ and that the kinetic constant of both reactions equals $2 \lambda$. Introduce further the variable $\xi=[A]-\frac{1}{2}$. Then the kinetic equation of the reaction system is given by $\frac{d \xi}{d t}=\lambda \xi-4 \lambda \xi^{3}$. A first order approximation of this kinetic equation at the unstable equilibrium $\xi=0$ gives $\frac{d \xi}{d t}=\lambda \xi$. Replacing $d \xi$ by $\mathbb{E}\left[\mathcal{Q}_{t} \mid \Xi=\xi_{t}\right]$ and $d t$ by 
$\delta t$ exhibits an analogy between the first order approximation and (27), i.e., the reaction steps of our interacting particle system can-in conditional expectation-be viewed as infinitesimal steps in a first order approximation (at $\xi=0$ ) of the dynamics of (11).

Thus the interacting particle system under consideration may be considered as a linearization of interacting particle systems modeling the spatio-temporal behavior of more complex chemical reactions. We do not further dwell on the question of linearization of more complex models in this article.

3.3 Remark Before we start with our introduction to nonstandard analysis, the formulation of the exact hypotheses for our extended model etc., we outline the main steps of our investigation that lead to the proof of our main results, the Theorems 6.5 and 3.1.

The Hypotheses 5.1 and 5.3 give us the stochastic model under consideration. It is a discrete time Markov process $\left(\Xi_{0}, \ldots, \Xi_{t}, \Xi_{t^{+}}, \ldots, \Xi_{T}\right)$. However, by the use of nonstandard analysis, our model may be considered as quasi-continuous.

By Proposition 5.4 and Definition 5.6 we split the short term evolution of our process $\Xi$ into a conditional deterministic and a pure random part, summarized in Remark 5.7 by the formula:

$$
\Xi_{t^{+}}=(1+\lambda \delta t) \Xi_{t}+\Sigma_{t} \text { and } \Xi_{t+\delta t}=\left(i d+\delta t \triangle_{h}\right) \Xi_{t^{+}}+\Sigma_{t^{+}}
$$

with $\Sigma_{t}$ and $\Sigma_{t^{+}}$random variables possessing expectation 0 .

In Proposition 5.9 the conditional covariance $\mathbb{E}\left[\left\langle\Sigma_{t} \mid \eta_{1}\right\rangle\left\langle\Sigma_{t} \mid \eta_{2}\right\rangle \mid \Xi_{t}=\xi_{t}\right]$ of the projections of $\Sigma$ onto directions $\eta_{1}$ and $\eta_{2}$ is investigated. In Proposition 5.11 the same is done for the conditional variance $\mathbb{E}\left[\left\langle\Sigma_{t^{+}} \mid \eta\right\rangle^{2} \mid \Xi_{t^{+}}=\xi_{t^{+}}\right]$. These investigations lead to the insight that mutually orthogonal projections of the random variables $\Sigma_{t}$ show almost independent behavior, while the variables $\Sigma_{t^{+}}$are rather small. Consequently it seems obvious to expand the system with respect to an orthonormal basis. Since the dynamics involves $\left(i d+\delta t \triangle_{h}\right) \Xi_{t^{+}}$an eigenbasis of the Laplace operator should be a good choice.

Thus we describe the systems dynamics in Remark 5.13 with respect to such an eigenbasis $\left(g_{k}\right)_{k \in \mathcal{K}}$ as

$$
\Xi_{t+\delta t}=\sum_{k \in \mathcal{K}} \Xi_{t+\delta t, k} g_{k}=\sum_{k \in \mathcal{K}}\left(1+\delta t \mu_{k}\right)\left((1+\lambda \delta t) \Xi_{t, k}+\Sigma_{t, k}\right) g_{k}+\Sigma_{t^{+}, k} g_{k} .
$$


Letting $\widetilde{\Sigma}_{t, k}:=\left(1+\delta t \mu_{k}\right) \Sigma_{t, k}+\Sigma_{t^{+}, k}$ we obtain by recursion in Proposition 6.11 that

$$
\Xi_{t, k}=\sum_{s \in[0 \ldots t)}\left(\prod_{u \in(s \ldots t)}(1+\lambda \delta u)\left(1+\mu_{k} \delta u\right)\right) \widetilde{\Sigma}_{s, k} .
$$

By rescaling the random variables $\widetilde{\Sigma}_{s}:=\sum \widetilde{\Sigma}_{s, k} g_{k}$ by linear transformations $\Phi_{s}$ (with approximately inverse transformations $\Phi_{-s}$, introduced in Definitions 6.2 and 6.3) the equality above can also be expressed by (see Proposition 6.11):

$$
\Xi_{t}=\Phi_{-t}\left(\Gamma_{t}\right) \quad \text { with } \quad \Gamma_{t}:=\sum_{s \in[0 \ldots t)} \delta \Gamma_{s} \quad \text { and } \quad \delta \Gamma_{s}:=\Phi_{s}\left(\widetilde{\Sigma}_{s}\right)
$$

Note that the operators $\Phi_{-t}$ are defined in such a way, that - for $\delta t=\delta$ independent of $t$ and $\lambda^{2} \delta$ infinitesimally small—we obtain (compare with Proposition 6.4)

$$
\left(\Phi_{-t} \circ \Gamma_{T}\right)_{k}=\oslash e^{\lambda(t-T)} e^{\mu_{k} t}(1+\oslash) \Gamma_{T, k},
$$

i.e., $t \mapsto\left(\Phi_{-t} \circ \Gamma_{T}\right)_{k}$ shows approximately exponential growth with rate $\lambda+\mu_{k}$ and $\Xi_{T, k}=\oslash e^{\mu_{k} T}(1+\oslash) \Gamma_{T, k}$. (The symbol $\oslash$ is introduced in Notation 4.6.)

The Proposition 5.17 is obtained from the Propositions 5.9 and 5.11 via the Propositions 5.14 and 5.15. Proposition 5.17 shows that the random variables $\widetilde{\Sigma}_{t, k}$ and $\widetilde{\Sigma}_{t, j}$ are for $k \neq j$ almost independent, possess expectation 0 and possesses approximately a variance of $\frac{\lambda}{2} \frac{\delta t}{h N}$. From this we derive the formula (77) for the conditional variances of $\delta \Gamma_{s}$. We show in Lemma 6.14 that

$$
\frac{\lambda}{2} \frac{\delta}{h N} \sum_{s \in[0 \ldots \nu / \lambda)} \frac{(1+\lambda \delta)^{2(T-s) / \delta}}{\left(1+\mu_{k} \delta\right)^{2 s / \delta}}={ }_{\oslash}(1+\oslash) \frac{e^{2 \lambda T}}{4 h N}
$$

This is applied (in the proof of Lemma 6.15) to sum the conditional variances of $\delta \Gamma_{s}$ given by equation (77) in Proposition 6.12. Since the random variables $\delta \Gamma_{s}$ are for $t \neq s$ independent, we know from Theorem A.7 that the random variables $\Gamma_{t}$ are for sufficiently large $t$ approximately normally distributed. Altogether we obtain by the scaling $e^{2 \lambda T}=4 h N(1+\oslash)$ that $\Gamma_{t} \sim_{\oslash} N\left(0, i d_{\mathcal{K}}\right)$ for any $t$ in $[\nu / \lambda \ldots T]$ with $\nu$ unlimited.

It finally remains to prove that the path of our stochastic process $\Xi$ stay almost surely infinitesimally close to 0 on the near interval $[0 \ldots T-\nu / \lambda]$ and that they follow almost surely the deterministic trajectories $\left[\Phi_{-t} \circ \Gamma_{T}\right](\omega)$ on the near interval $[\nu / \lambda \ldots T]$, i.e., to prove formulas (66) and (65) in Theorem 6.5 (and thus (10) and (9) in Theorem 3.1). This aim is achieved by application of the Doob inequality and use of the linear transformations $\Phi_{-t}$ in the second step of the proof of Lemma 6.15 and at the end of Section 6. While (66) and (10) bound the absolute deviation of stochastic paths 
from 0 , the inequalities (65) and (9) bound the relative deviations of stochastic paths from deterministic trajectories.

Note that (65) and (12) together imply that $t \mapsto \Xi_{t, k}(\omega)$ behaves for almost all $\omega$ and $t \in[\nu / \lambda \ldots T]$ approximately like $t \mapsto e^{\left(\lambda+\mu_{k}\right)(t-T)} e^{\mu_{k} T} \cdot \Gamma_{T, k}(\omega)$, i.e., $t \mapsto \Xi_{t, k}(\omega)$ shows approximately exponential growth with rate $\lambda+\mu_{k}$.

So, to understand the main ideas of the article, one has to decompose the system's dynamics with respect to an eigenbasis $\left(g_{k}\right)_{k \in \mathcal{K}}$ of the Laplacian $\Delta$, to admit formula (54), to have a look at the derivation of (77) from (54) in the proof of Proposition 6.12, and the derivation of Lemma 6.14. Going trough the first part of the proof of Lemma 6.15 one concludes (80)-(83) from Proposition 6.12, Lemma 6.14 and Theorem A.7. One proceeds with the second part of the proof of Lemma 6.15 that shows (65). Theorem 6.5 finally follows by some further simple computations.

\section{Preliminaries}

The notation and argumentation used in this article is supplied by the axiomatic system IST (see Nelson [18] or F and M Diener [8] and Kanovei and Reeken [13, Chapter 3]) that provides, beside the binary ZFC-predicate $\in$, also an unary predicate $s t$.) called standard. The results and arguments used in this paper however remain valid in other approaches to nonstandard analysis as well.

The reader familiar with a model theoretic approach (as found in Robinson [21], Stroyan and Bayod [24] or Lindstrøm [16]), or the axiom system HST [13, Chapter 1], has to keep in mind that the plain term set is used synonymously with the term internal set and that we work within one single model. We do not use $\mathrm{a}^{*}$-operation and denote by $\mathbb{N}$

and $\mathbb{R}$ the standard sets of all natural and real numbers, i.e., the sets $\mathbb{N}$ and $\mathbb{R}$ contain standard as well as nonstandard elements.

The reader new to nonstandard analysis is advised to have a look at the first pages of [18] or [8] to make himself familiar with the notions of standard, internal and external formula, the principles of transfer and idealization and some elementary consequences thereof.

To keep notation simple we write $\forall^{s t} x \phi(x)$ instead of $\forall x(s t(x) \Rightarrow \phi(x))$ and $\exists^{s t} x \phi(x)$ instead of $\exists x(s t(x) \wedge \phi(x))$. Given a set $M$ we use $x \in \underline{M}$ as shorthand for $x \in M \wedge \operatorname{st}(x)$ and $x \in \bar{M}$ as shorthand for $x \in M \wedge \neg s t(x)$. 
4.1 Notation Let $(X,\|\|$.$) be a normed space. We say that x \in(X,\|\|$.$) is limited$ and write $\|x\| \ll+\infty$ if $\exists^{s t} n \in \mathbb{N}$ such that $\|x\|<n$; otherwise, we say that $x$ is unlimited. In the case that $(X,\|\|)=.(\mathbb{R},||$.$) we also write -\infty \ll x \ll+\infty$ instead of $\|x\| \ll+\infty$. For positive unlimited $r \in(\mathbb{R},||$.$) we write r \approx \infty$. We say that $x \in(X,\|\|$.$) is infinitely small or infinitesimal if \forall^{s t} \varepsilon>0\|x\|<\varepsilon$. If $x-x^{\prime}$ is infinitely small we write $x \approx x^{\prime}$. Thus if $x$ is infinitely small we write $x \approx 0$. We say that $x \in \mathbb{R}$ is appreciable if it is limited but not infinitesimal. We call a set hyperfinite if it is finite and of unlimited (=hyperfinite) cardinality. Note that all the concepts introduced above are external.

We state some elementary results and definitions that can be obtained in IST without the axiom of standardization.

4.2 Definition Let $(Y,\|\|$.$) be a normed space. We say that the sequence$

$$
\left(y_{n}\right)_{n \in \mathbb{N}} \in Y^{\mathbb{N}} S \text {-converges to } y_{\infty} \in(Y,\|.\|), \quad \text { iff } \forall \nu \in \overline{\mathbb{N}} y_{\nu} \approx y_{\infty} .
$$

Let $Z$ be a subset of a normed space $(X,\|\|$.$) . We say that a function f: Z \rightarrow Y$ is uniformly S-continuous, if

$$
\forall z, z_{0} \in Z \wedge z \approx z_{0} \Rightarrow f(z) \approx f\left(z_{0}\right) .
$$

4.3 Remark A standard sequence $\left(x_{n}\right)_{n \in \mathbb{N}} \mathrm{S}$-converges if and only if there exists a standard $x_{\infty}$ such that $\left(x_{n}\right)_{n \in \mathbb{N}}$ converges (in the usual ZFC-based sense) to $x_{\infty}$. A standard function $f$ is uniformly $S$-continuous if and only if it is uniformly continuous in the usual sense. Both assertions follow from the permanence principle (e.g. Van den Berg [5, Chapter IV, Section 1]) and transfer. Further a bounded standard function is limited.

4.4 Definition (Compare with [19, first paragraph of Chapter 6].) Let $t_{0}, T \in \mathbb{R}$ be limited. A near interval $\left[t_{0} \ldots T\right]$ is a finite subset of $\left[t_{0}, T\right]$ such that $\left\{t_{0}, T\right\} \subset$ $\left[t_{0} \ldots T\right]$ and the distance of consecutive elements is infinitesimally small. We denote by $t+\delta t \in\left[t_{0} \ldots T\right]$ the successor of $t \in\left[t_{0} \ldots T\right]$ with respect to the usual ordering $\leq$ on $\left[t_{0} \ldots T\right]$. We say that the near interval $[0 \ldots T]$ is equally spaced if $\delta t$ is a

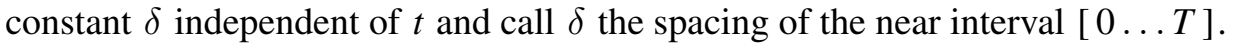

We let $\left[t_{0} \ldots T\right):=\left[t_{0} \ldots T\right] \backslash\{T\},\left(t_{0} \ldots T\right]:=\left[t_{0} \ldots T\right] \backslash\left\{t_{0}\right\}$ and $\left(t_{0} \ldots T\right):=$ $\left[t_{0} \ldots T\right] \backslash\left\{t_{0}, T\right\}$.

4.5 Remark It is convenient to use in some steps (Lemma 6.14) of the proof of Theorem 6.5 (and thus also in its statement) an equally spaced near interval. However, 
we use general near intervals in the formulation of Theorem A.7 and some other results, since such a formulation may turn out to be useful for further applications. Note that if we speak of a near interval $\left[t_{0} \ldots T\right]$ we presuppose the limitedness of $t_{0}$ and $T$.

4.6 Notation The domain and the range of a function $F$ is denoted by $\operatorname{dom}(F)$ and $\operatorname{ran}(F)$. We further introduce the symbol $\oslash$ and the notations $\leq_{\oslash}$ and $=_{\varnothing}$. We use them to handle calculations with non explicitly stated infinitesimal quantities, which simplify our notational effort. Let $F(x)$ and $G(y)$ denote functions of the variables $x$ and $y$. We define

$$
\begin{aligned}
F(\oslash) \leq_{\oslash} G(\oslash): & \Longleftrightarrow \\
& (\forall o \in \operatorname{dom}(F) o \approx 0 \Rightarrow \exists \hat{o} \in \operatorname{dom}(G) \hat{o} \approx 0 \wedge F(o) \leq G(\hat{o})) .
\end{aligned}
$$

The symbol $\oslash$ is used in the same manner if the character $\leq$ in (13) is replaced by the character $=$, i.e.,

$$
\begin{aligned}
F(\oslash)={ }_{\odot} G(\oslash): & \Longleftrightarrow \\
& (\forall o \in \operatorname{dom}(F) o \approx 0 \Rightarrow \exists \hat{o} \in \operatorname{dom}(G) \hat{o} \approx 0 \wedge F(o)=G(\hat{o})) .
\end{aligned}
$$

Note that $={ }_{\oslash}$ is not symmetric. (For example, we have $\rho={ }_{\oslash} \oslash$ for any infinitesimal $\rho$, but $\oslash \neq \varnothing \rho$ for any $\rho \in \mathbb{R}$.) Our definitions imply that

$$
\begin{gathered}
F(\oslash) \leq_{\oslash} G(\oslash) \wedge G(\oslash) \leq_{\oslash} H(\oslash) \Rightarrow F(\oslash) \leq_{\oslash} H(\oslash) \\
F(\oslash) \leq_{\oslash} G(\oslash) \Rightarrow F(\oslash)+H(\oslash) \leq_{\oslash} G(\oslash)+H(\oslash)
\end{gathered}
$$

and

$$
\left(\forall^{s t} \varepsilon>0\right)\left(\oslash \leq_{\oslash} \varepsilon\right) \text {. }
$$

For a different definition of the symbol $\oslash$ leading to the same use in calculus see Koudjeti and Van den Berg [14].

\section{Discrete Fourier Analysis and the Laplacian}

We will make use of the following well-known results from discrete Fourier analysis. For more information on the topic of discrete Fourier Analysis consult Terras [25] or Luong [17].

4.7 Definition Let $h$ be such that $1 / h \in \mathbb{N}$. Let $\mathcal{H}:=h \mathbb{Z} / \mathbb{Z}$. Let $\mathcal{K}(h):=\{k \in$ $\left.\mathbb{Z} \mid-\frac{1}{2 h}+\frac{1}{2} \leq k \leq \frac{1}{2 h}\right\}$ and let for $k \in \mathcal{K}(h)$ functions $g_{k} \in \mathbb{R}^{\mathcal{H}}$ be given by

$$
\begin{aligned}
& g_{k}(x):=\sqrt{h} \cos (2 \pi k x) \text { for } k=1 /(2 h), \text { provided that } 1 /(2 h) \in \mathbb{N} \\
& g_{k}(x):=\sqrt{2 h} \cos (2 \pi k x) \text { for } 1 /(2 h)>k>0 \\
& g_{k}(x):=\sqrt{2 h} \sin (2 \pi k x) \text { for } k<0 \text { and } \\
& g_{0}(x):=\sqrt{h} .
\end{aligned}
$$


4.8 Definition Let $\langle. \mid\rangle:. \mathbb{R}^{\mathcal{H}} \times \mathbb{R}^{\mathcal{H}} \rightarrow[0, \infty)$ denote the usual euclidean inner product given by $\langle f \mid g\rangle:=\sum_{x \in \mathcal{H}}[f \cdot g](x)$. We further define the 2-norm (euclidean norm) $\|.\|_{2}: \mathbb{R}^{\mathcal{H}} \rightarrow[0, \infty)$ by $\|f\|_{2}:=\sqrt{\langle f \mid f\rangle}$ and let $\mathbb{S}\left(\mathbb{R}^{\mathcal{H}}\right):=\left\{\xi \in \mathbb{R}^{\mathcal{H}} \mid\|\xi\|_{2}=1\right\}$ denote the unit sphere.

4.9 Notation We denote the imaginary unit by $i$ and the exponential function by $x \mapsto e^{x}$ or $x \mapsto \exp (x)$.

4.10 Remark Note that the family $\left(g_{k}\right)_{k \in \mathcal{K}(h)}$ of functions $g_{k} \in \mathbb{R}^{\mathcal{H}}$ defined in 4.7 forms an orthonormal bases of $\mathbb{R}^{\mathcal{H}}$ with respect to $\langle$.$\left.| . \right\rangle$, i.e., $\left\langle g_{k} \mid g_{l}\right\rangle=\epsilon_{k, l}$. This is most easily seen using the identities

$$
\cos (2 \pi k x)=\left[e^{2 \pi i k x}+e^{-2 \pi i k x}\right] / 2 \text { and } \sin (2 \pi k x)=\left[e^{2 \pi i k x}-e^{-2 \pi i k x}\right] / 2 i
$$

and that for $k, l \in \mathcal{K}(h)$ we have

$$
\left.h \sum_{x \in \mathcal{H}} e^{2 \pi i(k-l) x}=\epsilon_{k, l} \text { and } h \sum_{x \in \mathcal{H}} e^{2 \pi i(k+l) x}=\epsilon_{(k+l} \bmod 1 / h\right), 0
$$

4.11 Definition Let $1 / h \in \mathbb{N}$ and let $\mathcal{H}:=h \mathbb{Z} / \mathbb{Z}$. Define the discrete Laplace operator $\triangle_{h}: \mathbb{R}^{\mathcal{H}} \rightarrow \mathbb{R}^{\mathcal{H}}$ by $\left[\triangle_{h} f\right](x)=(f(x-h)+f(x+h)-2 f(x)) h^{-2}$.

4.12 Remark Note that the functions $g_{k}$ provided by Definition 4.7 are the eigenvectors of $\triangle_{h}$, i.e., $\triangle_{h} g_{k}=\mu_{k} g_{k}$. Further

$$
\mu_{k}=\mu_{-k}=\frac{2}{h^{2}}[\cos (2 \pi k h)-1] \leq 0 .
$$

This is most easily seen using the identity $e^{2 \pi i k x}=\cos (2 \pi k x)+i \sin (2 \pi k x)$ and calculating

$$
\begin{array}{r}
\triangle_{h} e^{2 \pi i k x}=\frac{1}{h^{2}}\left[e^{2 \pi i k(x+h)}+e^{2 \pi i k(x-h)}-2 e^{2 \pi i k x}\right] \\
=e^{2 \pi i k x} \frac{2}{h^{2}}[\cos (2 \pi k h)-1] .
\end{array}
$$

4.13 Remark For standard $k \in \mathcal{K}(h)$-and thus especially for any $k \in \mathcal{K}(h)$ provided that $h$ is standard-we have $-\infty \ll \mu_{k} \leq 0$. 


\section{Probabilities and Distributions}

4.14 Notation (Compare with Nelson [19, Chapter 1]) Our considerations will be based on a finite non-degenerate probability space $(\Omega, \mathcal{P})$, where $\Omega$ denotes a finite set and $\mathcal{P}$ a non-degenerate probability on $2^{\Omega}$, i.e., $\mathcal{P}: 2^{\Omega} \rightarrow[0,1]$ fulfills $\mathcal{P}\left(\Omega_{0}\right)=$ $\sum_{\omega \in \Omega_{0}} \mathcal{P}(\{\omega\}), \mathcal{P}(\Omega)=1$ and $\forall \omega \in \Omega \mathcal{P}(\{\omega\})>0$. We call a function $X$ a (partially defined) random variable if $\emptyset \neq \operatorname{dom}(X) \subseteq \Omega$. If $X$ and $Y$ are random variables we denote by $Y=y$ the set $\{\omega \mid Y(\omega)=y\}$ and let $\left.X\right|_{Y=y}$ denote the restriction of the function $X$ to $\operatorname{dom}(X) \cap\{\omega \mid Y(\omega)=y\}=: \operatorname{dom}\left(\left.X\right|_{Y=y}\right)$.

4.15 Definition (Compare with [19, Chapters 1 and 2]) Let $X$ and $Y$ be random variables. Let $\mathbb{P}(X=x):=\frac{\mathcal{P}(X=x)}{\mathcal{P}(\operatorname{dom}(X))}$. Note that $\operatorname{dom}(X)=\Omega$ implies that $\mathbb{P}(X=x)=\mathcal{P}(X=x)$. Given a function $f$ with $\operatorname{ran}(X) \subseteq \operatorname{dom}(f)$ and $\operatorname{ran}(f) \subseteq \mathbb{R}^{\mathcal{J}}$ (with $\mathcal{J}$ an arbitrary set), we let $\mathbb{E}[f \circ X]=\sum_{x} \mathbb{P}(X=x) f(x)$. In the case that $\operatorname{dom}\left(\left.X\right|_{Y=y}\right) \neq \emptyset$ we define by $\mathbb{P}(X=x \mid Y=y):=\mathbb{P}\left(\left.X\right|_{Y=y}=x\right)$ the conditional probability that $X=x$ under the hypothesis that $Y=y$. The conditional expectation $\mathbb{E}(f \circ X \mid Y=y)$ is defined by replacing the probabilities in the definition of the expectation above by conditional probabilities. Given a function $F$ with $\operatorname{ran}(Y) \subseteq \operatorname{dom}(F)$ we use $\mathbb{P}(X=x \mid Y)=F \circ Y$ as a shorthand notation for

$$
(\forall y \in \operatorname{ran}(Y))\left(\operatorname{dom}\left(\left.X\right|_{Y=y}\right) \neq \emptyset \Longrightarrow \mathbb{P}(X=x \mid Y=y)=F(y)\right) .
$$

4.16 Definition (Compare with [19, Chapters 3 and 9]) A stochastic process $X=$ $\left(X_{t}\right)_{t \in\left[t_{0} \ldots T\right]}$ with time $\left[t_{0} \ldots T\right]$ and state space $M$ is an indexed family of random variables $X_{t} \in M^{\Omega_{0}}$ with $\Omega_{0} \subseteq \Omega$. A stochastic process $X$ is Markov if for all $t \in$ $\left(t_{0} \ldots T\right)$ and all $\left(x_{s}\right)_{s \in\left[t_{0} \ldots t\right]} \in M^{\left[t_{0} \ldots t\right]}$ such that $\left(x_{s}\right)_{s \in\left[t_{0} \ldots t\right]} \in \operatorname{ran}\left(\left(X_{s}(\omega)\right)_{s \in\left[t_{0} \ldots t\right]}\right)$ we have

$$
\mathbb{P}\left(X_{t+\delta t}=x_{t+\delta t} \mid X_{t}=x_{t}\right)=\mathbb{P}\left(X_{t+\delta t}=x_{t+\delta t} \mid\left(X_{S}\right)_{s \in\left[t_{0} \ldots t\right]}=\left(x_{s}\right)_{s \in\left[t_{0} \ldots t\right]}\right)
$$

and it is a martingale if $M=\mathbb{R}^{\mathcal{J}}$ (for some arbitrary set $\mathcal{J}$ ) and

$$
s<t \wedge x_{s} \in \operatorname{ran}\left(X_{s}\right) \Rightarrow \mathbb{E}\left[X_{t} \mid X_{s}=x_{s}\right]=x_{s} .
$$

4.17 Definition Given a topological space $\mathcal{X}$, we denote by $\left(\mathcal{C}_{b}(\mathcal{X}),\|\cdot\|_{\infty}\right)$ the space of all bounded continuous functions $f: \mathcal{X} \rightarrow \mathbb{R}$ endowed with the $\|\cdot\|_{\infty}$-norm defined by $\|f\|_{\infty}:=\sup _{x \in \mathcal{X}}|f(x)|$. We further denote by $\mathcal{C}_{b}^{n}(\mathbb{R})$ the space of all $n$-times differentiable functions from $\mathbb{R}$ to $\mathbb{R}$ such that all derivatives (including the ${ }^{\text {th }}$ ) are continuous and bounded functions. We let $\mathcal{C}_{b}^{\infty}(\mathbb{R}):=\bigcap_{n \in \mathbb{N}} \mathcal{C}_{b}^{n}(\mathbb{R})$. 
4.18 Definition (Compare with Weisshaupt [28, Definition 3.3]) Let $Y: \Omega \rightarrow \mathbb{R}$ and let $\sigma \in(0, \infty)$ be limited. We say that the random variable $Y$ is approximately $N\left(0, \sigma^{2}\right)$ distributed and write $Y \sim_{\oslash} N\left(0, \sigma^{2}\right)$, if

$$
\left(\forall^{s t} f \in \mathcal{C}_{b}(\mathbb{R})\right) \quad\left(\mathbb{E}[f \circ Y] \approx \int_{y \in \mathbb{R}} f(y) \frac{\exp \left(-y^{2} /\left(2 \sigma^{2}\right)\right)}{\sqrt{2 \pi} \sigma} d y\right)
$$

with $\int_{y \in \mathbb{R}}$ denoting the integral in the sense of Riemann.

4.19 Remark Note that in contrast to [28, Definition 3.3] our Definition 4.18 does not presuppose almost limitedness of the random variable $Y$ and we do not make use of the concept of almost limitedness in this article. (For a definition of the concept of almost limitedness see for example [28, Definition 3.2].) However, an approximately normally distributed random variable in the sense of Definition 4.18 is almost limited in the sense of [28, Definition 3.2].

4.20 Remark Let $Y$ be approximately $N(0,1)$ distributed and let $\rho \approx 0$. Then $\mathbb{P}\left(Y^{2} \leq \rho\right) \approx 0$.

4.21 Definition Let $\mathcal{J}$ be a finite set. We let $\mathbb{S}^{*}\left(\mathbb{R}^{\mathcal{J}}\right)$ denote the family of all functionals $\psi^{*}: \mathbb{R}^{\mathcal{J}} \rightarrow \mathbb{R}$ of the from $\psi^{*}(\xi)=\sum_{j \in \mathcal{J}} \psi_{j}\left\langle\xi \mid e_{j}\right\rangle$, with $\psi_{j} \in \mathbb{R}$ and $\sum_{j \in \mathcal{J}} \psi_{j}^{2}=1$.

4.22 Definition Let $\mathcal{J}$ be a finite set and let $X: \Omega \rightarrow \mathbb{R}^{\mathcal{J}}$. We say that the random variable $X$ is approximately $N(0, i d)$ distributed on $\mathbb{R}^{\mathcal{J}}$ and write $X \sim_{\oslash} N\left(0, i d_{\mathcal{J}}\right)$ or simply $X \sim_{\oslash} N(0, i d)$ if

$$
\left(\forall \psi^{*} \in \mathbb{S}^{*}\left(\mathbb{R}^{\mathcal{J}}\right)\right)\left(\psi^{*}(X) \sim_{\oslash} N(0,1)\right) .
$$

4.23 Remark Definition 4.22 is partially justified by Proposition B.6 in Appendix B. Note however that the finite set $\mathcal{J}$ is supposed to be standard in Proposition B.6, while this is not the case in Definition 4.22.

4.24 Remark Note that $X \sim_{\oslash} N(0, i d)$ is equivalent with

$$
\left(\forall^{s t} f \in \mathcal{C}_{b}(\mathbb{R})\right)\left(\forall \psi^{*} \in \mathbb{S}^{*}\left(\mathbb{R}^{\mathcal{J}}\right)\right)\left(\mathbb{E}\left[f \circ \psi^{*}(X)\right] \approx \int_{y \in \mathbb{R}} f(y) \frac{\exp \left(-y^{2} / 2\right)}{\sqrt{2 \pi}} d y\right)
$$

which is further equivalent with

$$
\begin{aligned}
\left(\forall^{s t} \varepsilon>0\right)\left(\forall^{s t} f \in \mathcal{C}_{b}(\mathbb{R})\right) & \left(\forall \psi^{*} \in \mathbb{S}^{*}\left(\mathbb{R}^{\mathcal{J}}\right)\right) \\
& \left|\mathbb{E}\left[f \circ \psi^{*}(X)\right]-\int_{y \in \mathbb{R}} f(y) \frac{\exp \left(-y^{2} / 2\right)}{\sqrt{2 \pi}} d y\right|<\varepsilon .
\end{aligned}
$$




\section{The Model}

5.1 Hypothesis Suppose that $N \in \overline{\mathbb{N}}, \lambda \approx \infty$ and that $h \in(0,1]$ is such that

$$
\begin{array}{ll}
\text { Let } & \mathcal{H}:=h \mathbb{Z} / \mathbb{Z}, \quad \mathcal{K}(h):=\left\{k \in \mathbb{Z} \mid-\frac{1}{2 h}+\frac{1}{2} \leq k \leq \frac{1}{2 h}\right\} \\
\text { and let } & \mathbb{X}:=\left\{\frac{m}{h N}-\frac{1}{2} \mid m \in \mathbb{N} \text { and } 1 \leq m \leq h N-1\right\}^{\mathcal{H}} \subset \mathbb{R}^{\mathcal{H}} .
\end{array}
$$

Let $[0 \ldots T]$ be a near interval ${ }^{5}$ such that

$$
\forall t \in[0 \ldots T] \quad \delta t \lambda h N \approx 0 .
$$

Let further $\left(g_{k}\right)_{k \in \mathcal{K}(h)}$ denote the eigenbasis of the discrete Laplace operator, introduced in Definition 4.7, and denote by $\mu_{k}$ the eigenvalue corresponding to $g_{k}$.

5.2 Remark Suppose that $N_{A, t}(\omega), N_{A, t^{+}}(\omega)$ denote the numbers of type-A particles introduced in Section 3. Then $\Xi_{t}(\omega)$ and $\Xi_{t^{+}}(\omega)$, provided by Definition 5.3 below, model the deviation of the concentration of type A particles from $\frac{1}{2}$, i.e., for $N_{A, t}(\omega), N_{A, t^{+}}(\omega) \in\{0, \ldots, h N\}^{\mathcal{H}}$ we have that

$$
\Xi_{t}(\omega)=\frac{N_{A, t}(\omega)}{h N}-\frac{1}{2} \text { and } \Xi_{t^{+}}(\omega)=\frac{N_{A, t^{+}}(\omega)}{h N}-\frac{1}{2}
$$

and the dynamics specified by (23) and (25) coincides via

$$
\mathcal{Q}_{t}(\omega)=\frac{Q_{A, t}(\omega)}{h N} \text { and } \mathcal{Q}_{t^{+}}(\omega)=\frac{Q_{A, t^{+}}(\omega)}{h N}
$$

with the dynamics given by (3), (4) and (7).

5.3 Hypothesis Let $\left(\Xi_{t}\right)_{t \in[0 \ldots T]}$ and $\left(\Xi_{t^{+}}\right)_{t \in[0 \ldots T)}$ be indexed families of random variables $\Xi_{t}, \Xi_{t^{+}}: \Omega \rightarrow \mathbb{R}^{\mathcal{H}}$ such that

$$
\Xi:=\left(\Xi_{0}, \Xi_{0^{+}}, \ldots, \Xi_{t}, \Xi_{t^{+}}, \ldots, \Xi_{T}\right)
$$

forms a Markov Chain. We specify this Markov chain by its transitions from $\Xi_{t}$ to $\Xi_{t^{+}}$ and $\Xi_{t^{+}}$to $\Xi_{t+\delta t}$ given by random variables $\mathcal{Q}_{t}$ and $\mathcal{Q}_{t^{+}}$respectively, i.e., we suppose that $\Xi_{0}(\omega)=0 \in \mathbb{R}^{\mathcal{H}}$ independent of $\omega$ and let

$$
\Xi_{t^{+}}:=\Xi_{t}+\mathcal{Q}_{t} \text { and } \Xi_{t+\delta t}:=\Xi_{t^{+}}+\mathcal{Q}_{t^{+}}
$$

\footnotetext{
${ }^{5}$ Note that this presupposes that $T$ is limited, although we are not going to make use of this fact before Section 6 .
} 
If $\xi_{t} \in \mathbb{X}$ and $\mathbb{P}\left(\Xi_{t}=\xi_{t}\right)>0$ we let $\mathcal{Q}_{t} \in\left\{0,-\frac{e_{x}}{h N},+\frac{e_{x}}{h N} \mid x \in \mathcal{H}\right\}^{\Omega}$ be such that

$$
\mathbb{P}\left(\mathcal{Q}_{t}= \pm \frac{e_{x}}{h N} \mid \Xi_{t}=\xi_{t}\right)=p_{\lambda, t}^{ \pm}\left(\xi_{t}, x\right):=\delta t \frac{\lambda}{2} h N\left(\frac{1}{2} \pm \xi_{t}(x)\right)
$$

If $\xi_{t} \in \mathbb{R}^{\mathcal{H}} \backslash \mathbb{X}, \mathbb{P}\left(\Xi_{t}=\xi_{t}\right)>0$ let $\mathcal{Q}_{t} \in\left\{\delta t \lambda \xi_{t}, \delta t \lambda \xi_{t}-\frac{e_{x}}{h N}, \delta t \lambda \xi_{t}+\frac{e_{x}}{h N} \mid x \in \mathcal{H}\right\}^{\Omega}$ be such that

$$
\mathbb{P}\left(\mathcal{Q}_{t}=\delta t \lambda \xi_{t} \pm \frac{e_{x}}{h N} \mid \Xi_{t}=\xi_{t}\right)=p_{\lambda, t}^{ \pm}\left(\xi_{t}, x\right):=\delta t \frac{\lambda}{4} h N .
$$

If $\xi_{t^{+}} \in \mathbb{X}$ and $\mathbb{P}\left(\Xi_{t^{+}}=\xi_{t^{+}}\right)>0$ let $\mathcal{Q}_{t^{+}} \in\left\{0, \frac{-e_{x}+e_{x+h}}{h N}, \frac{-e_{x}+e_{x-h}}{h N} \mid x \in \mathcal{H}\right\}^{\Omega}$ be such that

$$
\begin{aligned}
& \mathbb{P}\left(\mathcal{Q}_{t^{+}}=\frac{-e_{x}+e_{x \pm h}}{h N} \mid \Xi_{t^{+}}=\xi_{t^{+}}\right)=p_{\triangle, t}^{ \pm}\left(\xi_{t^{+}}, x\right) \\
& \quad:=\frac{\delta t}{h^{2}} h N\left(\frac{1}{2}+\xi_{t^{+}}(x)\right)\left(\frac{1}{2}-\xi_{t^{+}}(x \pm h)\right) \leq \frac{1}{4} \frac{\delta t}{h^{2}} h N .
\end{aligned}
$$

If $\xi_{t^{+}} \in \mathbb{R}^{\mathcal{H}} \backslash \mathbb{X}$ and $\mathbb{P}\left(\Xi_{t^{+}}=\xi_{t^{+}}\right)>0$ we define $\mathcal{Q}_{t^{+}}$by

$$
\left.\mathcal{Q}_{t^{+}}\right|_{t^{+}}=\xi_{t^{+}}:=\delta t \triangle_{h} \xi_{t^{+}} .
$$

5.4 Proposition Suppose that the Hypotheses 5.1 and 5.3 are fulfilled. For $\xi_{t}, \xi_{t^{+}} \in \mathbb{R}^{\mathcal{H}}$ with $\mathbb{P}\left(\Xi_{t}=\xi_{t}\right)>0, \mathbb{P}\left(\Xi_{t^{+}}=\xi_{t^{+}}\right)>0$ we have:

$$
\begin{aligned}
\mathbb{E}\left[\mathcal{Q}_{t} \mid \Xi_{t}=\xi_{t}\right] & =\delta t \lambda \xi_{t} \\
\mathbb{E}\left[\mathcal{Q}_{t^{+}} \mid \Xi_{t^{+}}=\xi_{t^{+}}\right] & =\delta t \triangle_{h} \xi_{t^{+}}
\end{aligned}
$$

Proof In the case that $\xi_{t} \in \mathbb{R}^{\mathcal{H}} \backslash \mathbb{X}$ equation (27) is immediately derived from (24), since in this case $p_{\lambda, t}^{+}(\xi, x)=p_{\lambda, t}^{-}(\xi, x)$. If $\xi_{t} \in \mathbb{X}$ then (27) holds since by (23)

$$
\mathbb{E}\left[\mathcal{Q}_{t} \mid \Xi_{t}=\xi_{t}\right](x)=\frac{1}{h N} p_{\lambda, t}^{+}\left(\xi_{t}, x\right)-\frac{1}{h N} p_{\lambda, t}^{-}\left(\xi_{t}, x\right)=\delta t \lambda \xi_{t}(x) .
$$

Thus (27) holds for any $\xi_{t} \in \mathbb{R}^{\mathcal{H}}$. In the case that $\xi_{t^{+}} \in \mathbb{R}^{\mathcal{H}} \backslash \mathbb{X}$ equation (28) is a consequence of (26). Finally if $\xi_{t^{+}} \in \mathbb{X}$ we obtain from (25) that

$$
\begin{aligned}
& \mathbb{E}\left[\mathcal{Q}_{t^{+}} \mid \Xi_{t^{+}}=\xi_{t^{+}}\right](x) \\
& =\frac{\delta t}{h^{2}}\left[-\left(\frac{1}{2}+\xi_{t^{+}}(x)\right)\left(\frac{1}{2}-\xi_{t^{+}}(x+h)\right)-\left(\frac{1}{2}+\xi_{t^{+}}(x)\right)\left(\frac{1}{2}-\xi_{t^{+}}(x-h)\right)\right. \\
& \left.\quad \quad+\left(\frac{1}{2}-\xi_{t^{+}}(x)\right)\left(\frac{1}{2}+\xi_{t^{+}}(x+h)\right)+\left(\frac{1}{2}-\xi_{t^{+}}(x)\right)\left(\frac{1}{2}+\xi_{t^{+}}(x-h)\right)\right] \\
& =\frac{\delta t}{h^{2}}\left(\xi_{t^{+}}(x-h)+\xi_{t^{+}}(x+h)-2 \xi_{t^{+}}(x)\right)=\delta t \triangle_{h} \xi_{t^{+}}(x) .
\end{aligned}
$$

Thus (28) has been shown for any $\xi_{t^{+}} \in \mathbb{R}$. 
5.5 Remark We may associate with the stochastic dynamical system fulfilling the Hypotheses 5.1 and 5.3 a deterministic system given by:

$$
\xi_{t^{+}}=\xi_{t}+\delta t \lambda \xi_{t} \text { and } \xi_{t+\delta t}=\xi_{t^{+}}+\delta t \triangle_{h} \xi_{t^{+}}
$$

While the increments of the stochastic system are given by $\mathcal{Q}_{t}$ and $\mathcal{Q}_{t^{+}}$the increments of the deterministic system (29) coincide (by Proposition 5.4) in the cases that $\mathbb{P}\left(\Xi_{t}=\right.$ $\left.\xi_{t}\right)>0, \mathbb{P}\left(\Xi_{t^{+}}=\xi_{t^{+}}\right)>0$ with the conditional expectations $\mathbb{E}\left[Q_{t} \mid \Xi_{t}=\xi_{t}\right]$ and $\mathbb{E}\left[Q_{t^{+}} \mid \Xi_{t^{+}}=\xi_{t^{+}}\right]$. Note further that $\xi=0$ is an equilibrium of the associated deterministic system, i.e., $\xi_{t}=0 \Rightarrow \xi_{t+\delta t}=0$. For standard $h$ and unlimited $\lambda$ the equilibrium $\xi=0$ is unique and unstable, since $\left\|\xi_{t+\delta t}\right\|_{2} \geq\left(1+\delta t \frac{\lambda}{2}\right)\left\|\xi_{t}\right\|_{2}$. This last fact follows from an expansion of the dynamics with respect to the eigenbasis $\left(g_{k}\right)_{k \in \mathcal{K}(h)}$ of $\Delta_{h}$ provided by ${ }^{6}$

$$
\xi_{t+\delta t}=\sum_{k \in \mathcal{K}(h)} \xi_{t+\delta t, k} g_{k}=(1+\delta t \lambda)\left(1+\delta t \mu_{k}\right) \xi_{t, k} g_{k}
$$

that implies, using $-\infty \ll \mu_{k}(\operatorname{Remark} 4.13$ and $1 / h \in \underline{\mathbb{N}})$ and $\lambda \approx+\infty$,

$$
\begin{aligned}
\left\|\xi_{t+\delta t}\right\|_{2}^{2}= & \sum_{k \in \mathcal{K}(h)} \xi_{t+\delta t, k}^{2} \geq \sum_{k \in \mathcal{K}(h)}(1+\delta t \lambda)^{2}\left(1+\delta t \mu_{k}\right)^{2} \xi_{t, k}^{2} \\
& \geq \sum_{k \in \mathcal{K}(h)}(1+\delta t \lambda / 2)^{2} \xi_{t, k}^{2}=(1+\delta t \lambda / 2)^{2}\left\|\xi_{t}\right\|_{2}^{2} .
\end{aligned}
$$

5.6 Definition To investigate $\Xi_{t}$ further we define:

$$
\begin{gathered}
\Sigma_{t}:=\mathcal{Q}_{t}-\mathbb{E}\left[\mathcal{Q}_{t} \mid \Xi_{t}\right] \stackrel{(a)}{=} \mathcal{Q}_{t}-\delta t \lambda \Xi_{t} \\
\Sigma_{t^{+}}:=\mathcal{Q}_{t^{+}}-\mathbb{E}\left[\mathcal{Q}_{t^{+}} \mid \Xi_{t^{+}}\right] \stackrel{(b)}{=} \mathcal{Q}_{t^{+}}-\delta t \triangle_{h} \Xi_{t^{+}}
\end{gathered}
$$

5.7 Remark Note that the equalities (a) and (b) in (30) and (31) follow from (27) and (28) respectively. From (22), (30) and (31) we obtain that:

$$
\Xi_{t^{+}}=(1+\lambda \delta t) \Xi_{t}+\Sigma_{t} \text { and } \Xi_{t+\delta t}=\left(i d+\delta t \triangle_{h}\right) \Xi_{t^{+}}+\Sigma_{t^{+}}
$$

5.8 Remark As a consequence of Definition 5.6 we obtain for all $\xi_{t}, \xi_{t^{+}} \in \mathbb{R}^{\mathcal{H}}$ with $\mathbb{P}\left(\Xi_{t}=\xi_{t}\right)>0, \mathbb{P}\left(\Xi_{t^{+}}=\xi_{t^{+}}\right)>0$ that

$$
\mathbb{E}\left[\Sigma_{t} \mid \Xi_{t}=\xi_{t}\right]=0, \quad \mathbb{E}\left[\Sigma_{t^{+}} \mid \Xi_{t^{+}}=\xi_{t^{+}}\right]=0
$$

Further for all $\omega \in \Omega$ we obtain from Definition 5.6 and Definition 5.3 that:

$$
\left\|\Sigma_{t}(\omega)\right\|_{2} \leq \frac{2}{h N},\left\|\Sigma_{t^{+}}(\omega)\right\|_{2} \leq \sqrt{2} \frac{2}{h N}
$$

\footnotetext{
${ }^{6}$ Compare with equation (44) in Remark 5.13.
} 
5.9 Proposition Suppose that the Hypotheses 5.1 and 5.3 are fulfilled and suppose that $\eta_{1}, \eta_{2} \in \mathbb{S}\left(\mathbb{R}^{\mathcal{H}}\right), \xi_{t} \in \mathbb{R}^{\mathcal{H}}$ and $\mathbb{P}\left(\Xi_{t}=\xi_{t}\right)>0$. Then

$$
\mathbb{E}\left[\left\langle\mathcal{Q}_{t} \mid \eta_{1}\right\rangle \mid \Xi_{t}=\xi_{t}\right]=\left\langle\mathbb{E}\left[\mathcal{Q}_{t} \mid \Xi_{t}=\xi_{t}\right] \mid \eta_{1}\right\rangle=\delta t \lambda\left\langle\xi_{t} \mid \eta_{1}\right\rangle
$$

For $\Sigma_{t}$ given by (30) we obtain

$$
\mathbb{E}\left[\left\langle\Sigma_{t} \mid \eta_{1}\right\rangle\left\langle\Sigma_{t} \mid \eta_{2}\right\rangle \mid \Xi_{t}=\xi_{t}\right]={ }_{\oslash} \frac{\lambda}{2} \frac{\delta t}{h N}\left(\left\langle\eta_{1} \mid \eta_{2}\right\rangle+\oslash\right)
$$

in the case that $\xi_{t} \in \mathbb{X}$ (which implies that $\xi_{t}$ is limited) and also in the case $\xi_{t} \in \mathbb{R}^{\mathcal{H}} \backslash \mathbb{X}$.

Proof (35) holds by (27) and because the finite sums involved in the calculation of the conditional expectation $\mathbb{E}\left[. \mid \Xi_{t}=\xi_{t}\right]$ and the inner product $\left\langle. \mid \eta_{1}\right\rangle$ interchange. We prove (36) for $\xi_{t} \in \mathbb{X}$ first. From (23) we obtain

$$
\mathbb{P}\left(\mathcal{Q}_{t}^{2}=\frac{e_{x}}{h^{2} N^{2}} \mid \Xi_{t}=\xi_{t}\right)=p_{\lambda, t}^{+}\left(\xi_{t}, x\right)+p_{\lambda, t}^{-}\left(\xi_{t}, x\right)=\delta t \frac{\lambda}{2} h N
$$

and thus further that

$$
\begin{array}{r}
\mathbb{E}\left[\left\langle\mathcal{Q}_{t} \mid \eta_{1}\right\rangle\left\langle\mathcal{Q}_{t} \mid \eta_{2}\right\rangle \mid \Xi_{t}=\xi_{t}\right]=\sum_{x \in \mathcal{H}} \mathbb{P}\left(\mathcal{Q}_{t}^{2}=\frac{e_{x}}{h^{2} N^{2}} \mid \Xi_{t}=\xi_{t}\right) \frac{\eta_{1}(x) \eta_{2}(x)}{h^{2} N^{2}} \\
=\frac{\lambda}{2} \frac{\delta t}{h N}\left\langle\eta_{1} \mid \eta_{2}\right\rangle .
\end{array}
$$

We derive (36) by calculating

$$
\begin{aligned}
\mathbb{E}\left[\left\langle\Sigma_{t} \mid \eta_{1}\right\rangle\right. & \left.\left\langle\Sigma_{t} \mid \eta_{2}\right\rangle \mid \Xi_{t}=\xi_{t}\right] \\
& \stackrel{(a)}{=} \mathbb{E}\left[\left\langle\mathcal{Q}_{t} \mid \eta_{1}\right\rangle\left\langle\mathcal{Q}_{t} \mid \eta_{2}\right\rangle \mid \Xi_{t}=\xi_{t}\right]-\mathbb{E}\left[\left\langle\mathcal{Q}_{t} \mid \eta_{1}\right\rangle \mid \Xi_{t}=\xi_{t}\right] \cdot \mathbb{E}\left[\left\langle\mathcal{Q}_{t} \mid \eta_{2}\right\rangle \mid \Xi_{t}=\xi_{t}\right] \\
& \stackrel{(b)}{=} \frac{\lambda}{2} \frac{\delta t}{h N}\left\langle\eta_{1} \mid \eta_{2}\right\rangle-\delta t^{2} \lambda^{2}\left\langle\xi_{t} \mid \eta_{1}\right\rangle\left\langle\xi_{t} \mid \eta_{2}\right\rangle \stackrel{(c)}{=} \frac{\lambda}{2} \frac{\delta t}{h N}\left(\left\langle\eta_{1} \mid \eta_{2}\right\rangle+\oslash\right)
\end{aligned}
$$

with (a) a consequence of (30), equality (b) concluded from (35), (38) and equality (c) implied by (20) and the fact that $\xi_{t} \in \mathbb{X}$ is limited.

In the case $\xi_{t} \in \mathbb{R}^{\mathcal{H}} \backslash \mathbb{X}$ we conclude $\mathbb{E}\left[\left\langle\Sigma_{t} \mid \eta_{1}\right\rangle\left\langle\Sigma_{t} \mid \eta_{2}\right\rangle \mid \Xi_{t}=\xi_{t}\right]=\frac{\lambda}{2} \frac{\delta t}{h N}\left\langle\eta_{1} \mid \eta_{2}\right\rangle$ using (30) by calculations analogous to (37) and (38) with $\mathcal{Q}_{t}$ replaced by $\Sigma_{t}$.

5.10 Remark Suppose that we are given a function $\eta \in \mathbb{R}^{\mathcal{H}}$. Then

$$
\sum_{x \in \mathcal{H}}\left\langle e_{x+h}-e_{x} \mid \eta\right\rangle^{2}=\sum_{x \in \mathcal{H}}\left\langle e_{x-h}-e_{x} \mid \eta\right\rangle^{2}
$$

and

$$
\mathbb{E}\left[\left\langle\mathcal{Q}_{t^{+}} \mid \eta\right\rangle \mid \Xi_{t^{+}}=\xi_{t^{+}}\right] \stackrel{(a)}{=}\left\langle\mathbb{E}\left[\mathcal{Q}_{t^{+}} \mid \Xi_{t^{+}}=\xi_{t^{+}}\right] \mid \eta\right\rangle \stackrel{(b)}{=} \delta t\left\langle\triangle_{h} \xi_{t^{+}} \mid \eta\right\rangle \stackrel{(c)}{=} \delta t\left\langle\xi_{t^{+}} \mid \triangle_{h} \eta\right\rangle
$$

(Equality (a) follows by the interchange of finite sums, equality (b) follows from (28) and equality (c) from the fact that $\triangle_{h}$ is symmetric, i.e., $\triangle_{h}$ acts on $\mathbb{R}^{\mathcal{H}}$ as a self-adjoint operator.) 
5.11 Proposition Suppose that the Hypotheses 5.1 and 5.3 are fulfilled. Let $\eta \in \mathbb{R}^{\mathcal{H}}$ and let $\Sigma_{t^{+}}$be given by (31). Suppose that $\xi_{t^{+}} \in \mathbb{X}$ and $\mathbb{P}\left(\Xi_{t^{+}}=\xi_{t^{+}}\right)>0$. Then

$$
\mathbb{E}\left[\left\langle\Sigma_{t^{+}} \mid \eta\right\rangle^{2} \mid \Xi_{t^{+}}=\xi_{t^{+}}\right] \leq \frac{1}{2} \frac{\delta t}{h N} \frac{1}{h} \int_{\mathbb{R} / \mathbb{Z}}\left(\widetilde{\eta}^{\prime}\right)^{2}(x) d x
$$

with $\widetilde{\eta}: \mathbb{R} / \mathbb{Z} \rightarrow \mathbb{R}$ denoting a differentiable extension of $\eta$ and $\widetilde{\eta}^{\prime}$ denoting the derivative of $\widetilde{\eta}$.

Proof We calculate for $\xi_{t} \in \mathbb{X}$

$$
\begin{aligned}
\mathbb{E}\left[\left\langle\Sigma_{t^{+}} \mid \eta\right\rangle^{2} \mid \Xi_{t^{+}}=\right. & \left.\xi_{t^{+}}\right] \leq \mathbb{E}\left[\left\langle\mathcal{Q}_{t^{+}} \mid \eta\right\rangle^{2} \mid \Xi_{t^{+}}=\xi_{t^{+}}\right] \\
& \stackrel{(a)}{\leq} \frac{1}{4} \frac{\delta t}{h N} \sum_{x \in \mathcal{H}}\left[\frac{1}{h^{2}}\left\langle e_{x+h}-e_{x} \mid \eta\right\rangle^{2}+\frac{1}{h^{2}}\left\langle e_{x-h}-e_{x} \mid \eta\right\rangle^{2}\right] \\
& \stackrel{(b)}{=} \frac{1}{2} \frac{\delta t}{h N} \sum_{x \in \mathcal{H}} \frac{1}{h^{2}}\left\langle e_{x+h}-e_{x} \mid \eta\right\rangle^{2}=\frac{1}{2} \frac{\delta t}{h N} \sum_{x \in \mathcal{H}}\left(\frac{\eta(x+h)-\eta(x)}{h}\right)^{2} \\
& \stackrel{(c)}{\leq} \frac{1}{2} \frac{\delta t}{h N} \frac{1}{h} \int_{0}^{1}\left(\widetilde{\eta}^{\prime}\right)^{2}(x) d x .
\end{aligned}
$$

Note that inequality (a) follows from (25), equality (b) from (39) and inequality (c) from C.2.

5.12 Definition Given a random variable $X: \Omega \rightarrow \mathbb{R}^{\mathcal{H}}$ we define random Fourier coefficients $X_{k}$ for $k \in \mathcal{K}(h)$ by series expansion of $X$ with respect to the basis $\left(g_{k}\right)_{k \in \mathcal{K}(h)}$, i.e., we let

$$
X=: \sum_{k \in \mathcal{K}(h)} X_{k} g_{k} \text { or equivalently } X_{k}:=\left\langle X \mid g_{k}\right\rangle .
$$

Replacing the letter $X$ in (41) by $\Xi_{t}, \Xi_{t^{+}}, \Sigma_{t}, \Sigma_{t^{+}}, \Gamma_{t}$ and $\left(\Phi_{-t} \circ \Gamma_{t}\right)$ we analogously define random coefficients $\Xi_{t, k}, \Xi_{t^{+}, k}, \Sigma_{t, k}, \Sigma_{t^{+}, k}, \Gamma_{t, k}$ and $\left(\Phi_{-t} \circ \Gamma_{t}\right)_{k}$ by series expansion of the random variables $\Xi_{t}, \Xi_{t^{+}}, \Sigma_{t}, \Sigma_{t^{+}}, \Gamma_{t}$ and $\left(\Phi_{-t} \circ \Gamma_{t}\right)$. (The random variables $\Gamma_{t}$ are introduced in Definition 6.3.)

5.13 Remark From (32) and Definition 5.12 we obtain that

$$
\Xi_{t^{+}, k} g_{k}=(1+\lambda \delta t) \Xi_{t, k} g_{k}+\Sigma_{t, k} g_{k}
$$

and from (32), Definition 5.12 and Remark 4.12 that

(43) $\Xi_{t+\delta t, k} g_{k}=\Xi_{t^{+}, k}\left(i d+\delta t \triangle_{h}\right) g_{k}+\Sigma_{t^{+}, k} g_{k}=\Xi_{t^{+}, k}\left(1+\delta t \mu_{k}\right) g_{k}+\Sigma_{t^{+}, k} g_{k}$.

From (42) and (43) we obtain that

$$
\Xi_{t+\delta t, k}=\left(1+\delta t \mu_{k}\right)\left((1+\lambda \delta t) \Xi_{t, k}+\Sigma_{t, k}\right)+\Sigma_{t^{+}, k} .
$$


5.14 Proposition Suppose that the Hypotheses 5.1 and 5.3 are fulfilled, that $\Sigma_{t}$ and $\Sigma_{t^{+}}$denote the random variables introduced in Definition 5.6 and that the subscript $k$ refers to coordinates with respect to the series expansion introduced in Definition 5.12. Then for $\xi_{t}, \xi_{t^{+}} \in \mathbb{R}^{\mathcal{H}}$, with $\mathbb{P}\left(\Xi_{t}=\xi_{t}\right)>0, \mathbb{P}\left(\Xi_{t^{+}}=\xi_{t^{+}}\right)>0$ we have:

$$
\begin{aligned}
\mathbb{E}\left[\left(\Sigma_{t^{+}, k}\right)^{2} \mid \Xi_{t^{+}}=\xi_{t^{+}}\right] & \leq \frac{1}{2} \frac{\delta t}{h N}(2 \pi k)^{2} \\
\mathbb{E}\left[\left(\Sigma_{t^{+}, k}\right)^{2} \mid \Xi_{t}=\xi_{t}\right] & \leq \frac{1}{2} \frac{\delta t}{h N}(2 \pi k)^{2}
\end{aligned}
$$

Proof From (40) we obtain that

$$
\begin{gathered}
\xi_{t^{+}} \in \mathbb{X} \Longrightarrow \mathbb{E}\left[\left(\Sigma_{t^{+}, k}\right)^{2} \mid \Xi_{t^{+}}=\xi_{t^{+}}\right]=\mathbb{E}\left[\left\langle\Sigma_{t^{+}} \mid g_{k}\right\rangle^{2} \mid \Xi_{t^{+}}=\xi_{t^{+}}\right] \\
\leq \frac{1}{2} \frac{\delta t}{h N} \frac{1}{h} \int_{\mathbb{R} / \mathbb{Z}}\left(\widetilde{g}_{k}^{\prime}\right)^{2}(x) d x \leq \frac{1}{2} \frac{\delta t}{h N}(2 \pi k)^{2}
\end{gathered}
$$

with $\widetilde{g}_{k}^{\prime}: \mathbb{R} / \mathbb{Z} \rightarrow \mathbb{R}$ given by

$$
\begin{aligned}
& \widetilde{g}_{k}^{\prime}(x)=-2 \pi k \sqrt{h} \sin (2 \pi k x) \text { for } k=1 /(2 h), \text { provided } 1 /(2 h) \in \mathbb{N} \\
& \widetilde{g}_{k}^{\prime}(x)=-2 \pi k \sqrt{2 h} \sin (2 \pi k x) \text { for } 1 /(2 h)>k>0 \\
& \widetilde{g}_{k}^{\prime}(x)=2 \pi k \sqrt{2 h} \cos (2 \pi k x) \text { for } k<0 \\
& \text { and } \widetilde{g}_{0}^{\prime}=0 .
\end{aligned}
$$

From (26) and (31) we obtain that

$$
\xi_{t^{+}} \in \mathbb{R}^{\mathcal{H}} \backslash \mathbb{X} \Longrightarrow \mathbb{E}\left[\left(\Sigma_{t^{+}, k}\right)^{2} \mid \Xi_{t^{+}}=\xi_{t^{+}}\right]=0
$$

and from(47) and (48) we conclude that (45) holds. Since

$$
\left(\Xi_{0}, \Xi_{0^{+}}, \ldots, \Xi_{t}, \Xi_{t^{+}}, \ldots, \Xi_{T}\right)
$$

is a Markov chain we obtain from (45) that

$$
\begin{array}{r}
\mathbb{E}\left[\left(\Sigma_{t^{+}, k}\right)^{2} \mid \Xi_{t}=\xi_{t}\right]=\sum_{\xi_{t} \in \Upsilon_{t}} \mathbb{P}\left(\Xi_{t^{+}}=\xi_{t^{+}} \mid \Xi_{t}=\xi_{t}\right) \cdot \mathbb{E}\left[\left(\Sigma_{t^{+}, k}\right)^{2} \mid \Xi_{t^{+}}=\xi_{t^{+}}\right] \\
\leq \frac{1}{2} \frac{\delta t}{h N}(2 \pi k)^{2}
\end{array}
$$

with $\Upsilon_{t}:=\left\{\xi_{t^{+}} \mid \mathbb{P}\left(\Xi_{t^{+}}=\xi_{t^{+}}\right)>0\right\}$, i.e., we obtain that (46) holds.

5.15 Proposition Suppose that the Hypotheses 5.1 and 5.3 are fulfilled, that $\Sigma_{t}$ and $\Sigma_{t^{+}}$denote the random variables introduced in Definition 5.6 and that the subscript $k$ 
refers to coordinates with respect to the series expansion introduced in Definition 5.12. Then for all $\xi_{t} \in \mathbb{R}$ with $\mathbb{P}\left(\Xi_{t}=\xi_{t}\right)>0$ we obtain:

$$
\begin{aligned}
\mathbb{E}\left[\Sigma_{t, j} \cdot \Sigma_{t, k} \mid \Xi_{t}\right. & \left.=\xi_{t}\right]={ }_{\oslash} \frac{\lambda}{2} \frac{\delta t}{h N}\left(\epsilon_{j, k}+\oslash\right) \\
\mathbb{E}\left[\Sigma_{t^{+}, j} \cdot \Sigma_{t^{+}, k} \mid \Xi_{t}\right. & \left.=\xi_{t}\right] \leq \frac{1}{2} \frac{\delta t}{h N}(2 \pi)^{2} j k \\
\mathbb{E}\left[\Sigma_{t, j} \cdot \Sigma_{t^{+}, k} \mid \Xi_{t}\right. & \left.=\xi_{t}\right] \leq \oslash \frac{\sqrt{\lambda}}{2} \frac{\delta t}{h N}(2 \pi k)(1+\oslash) .
\end{aligned}
$$

Proof We obtain from (36) that

$$
\begin{aligned}
\mathbb{E}\left[\Sigma_{t, j} \cdot \Sigma_{t, k} \mid \Xi_{t}=\xi_{t}\right] & =\mathbb{E}\left[\left\langle\Sigma_{t} \mid g_{j}\right\rangle \cdot\left\langle\Sigma_{t} \mid g_{k}\right\rangle \mid \Xi_{t}=\xi_{t}\right] \\
& ={ }_{\oslash} \frac{\lambda}{2} \frac{\delta t}{h N}\left(\left\langle g_{j} \mid g_{k}\right\rangle+\oslash\right)={ }_{\oslash} \frac{\lambda}{2} \frac{\delta t}{h N}\left(\epsilon_{j, k}+\oslash\right) .
\end{aligned}
$$

Thus (49) holds for any $\xi_{t} \in \mathbb{R}$. From (46) we obtain that

$$
\begin{aligned}
\mathbb{E}\left[\Sigma_{t^{+}, j} \cdot \Sigma_{t^{+}, k} \mid \Xi_{t}=\xi_{t}\right] & \leq \sqrt{\mathbb{E}\left[\left(\Sigma_{t^{+}, j}\right)^{2} \mid \Xi_{t}=\xi_{t}\right]} \sqrt{\mathbb{E}\left[\left(\Sigma_{t^{+}, k}\right)^{2} \mid \Xi_{t}=\xi_{t}\right]} \\
& \leq \frac{1}{2} \frac{\delta t}{h N}(2 \pi)^{2} j k .
\end{aligned}
$$

which proves (50). From (46) and (49) we obtain that

$$
\begin{aligned}
\mathbb{E}\left[\Sigma_{t, j} \cdot \Sigma_{t^{+}, k} \mid \Xi_{t}=\xi_{t}\right) & \leq \sqrt{\mathbb{E}\left[\Sigma_{t, j}^{2} \mid \Xi_{t}=\xi_{t}\right]} \sqrt{\mathbb{E}\left[\Sigma_{t^{+}, k}^{2} \mid \Xi_{t}=\xi_{t}\right]} \\
& \leq \oslash \frac{\sqrt{\lambda}}{2} \frac{\delta t}{h N}(2 \pi k)(1+\oslash) .
\end{aligned}
$$

which proves (51).

5.16 Definition Suppose that $\Sigma_{t}$ and $\Sigma_{t^{+}}$denote the random variables introduced in Definition 5.6 and that the subscript ${ }_{k}$ refers to coordinates with respect to the series expansion introduced in Definition 5.12. Let

$$
\widetilde{\Sigma}_{t, k}:=\left(1+\delta t \mu_{k}\right) \Sigma_{t, k}+\Sigma_{t^{+}, k} \text { and let } \widetilde{\Sigma}_{t}:=\sum_{k \in \mathcal{K}(h)} \widetilde{\Sigma}_{t, k} g_{k}
$$

5.17 Proposition Suppose that the Hypotheses 5.1 and 5.3 are fulfilled, that $\widetilde{\Sigma}_{t, k}$ and $\widetilde{\Sigma}_{t}$ denote the random variables introduced in Definition 5.16. Then for all $\omega \in \Omega$ and all $\xi_{t} \in \mathbb{R}$ with $\mathcal{P}\left(\Xi_{t}=\xi_{t}\right)>0$ we have:

$$
\begin{gathered}
\left\|\widetilde{\Sigma}_{t}(\omega)\right\|_{2} \leq \frac{8}{h N} \\
\mathbb{E}\left[\widetilde{\Sigma}_{t} \mid \Xi_{t}=\xi_{t}\right]=0 \in \mathbb{R}^{\mathcal{H}} \\
\mathbb{E}\left[\widetilde{\Sigma}_{t, j} \cdot \widetilde{\Sigma}_{t, k} \mid \Xi_{t}=\xi_{t}\right]={ }_{\oslash}\left(\epsilon_{j, k}+\oslash\right) \frac{\lambda}{2} \frac{\delta t}{h N}
\end{gathered}
$$


Proof Equation (52) is a consequence of (34), $-\infty \ll \mu_{k} \leq 0$ (Remark 4.13) and Definition 5.16, while (53) follows from (33) and Definition 5.16 by

$$
\begin{aligned}
\mathbb{E}\left[\widetilde{\Sigma}_{t, k} \mid \Xi_{t}=\xi_{t}\right] & =\mathbb{E}\left[\left(1+\delta t \mu_{k}\right) \Sigma_{t, k} \mid \Xi_{t}=\xi_{t}\right]+\mathbb{E}\left[\Sigma_{t^{+}, k} \mid \Xi_{t}=\xi_{t}\right] \\
& =0+\sum_{\xi_{t} \in \Upsilon_{t}} \mathbb{P}\left(\Xi_{t^{+}}=\xi_{t^{+}} \mid \Xi_{t}=\xi_{t}\right) \cdot \mathbb{E}\left[\left(\Sigma_{t^{+}, k}\right) \mid \Xi_{t^{+}}=\xi_{t^{+}}\right]=0
\end{aligned}
$$

with $\Upsilon_{t}:=\left\{\xi_{t^{+}} \mid \mathbb{P}\left(\Xi_{t^{+}}=\xi_{t^{+}}\right)>0\right\}$. We finally obtain (54) since

$$
\begin{aligned}
& \mathbb{E}\left[\widetilde{\Sigma}_{t, j} \cdot \widetilde{\Sigma}_{t, k} \mid\right.\left.\Xi_{t}=\xi_{t}\right] \\
&= \mathbb{E}\left[\left(\left(1+\delta t \mu_{j}\right) \Sigma_{t, j}+\Sigma_{t^{+}, j}\right) \cdot\left(\left(1+\delta t \mu_{k}\right) \Sigma_{t, k}+\Sigma_{t^{+}, k}\right) \mid \Xi_{t}=\xi_{t}\right] \\
& \stackrel{(a)}{=}(1+\oslash)\left(\mathbb{E}\left[\Sigma_{t, j} \cdot \Sigma_{t, k} \mid \Xi_{t}=\xi_{t}\right]+\mathbb{E}\left[\Sigma_{t, j} \cdot \Sigma_{t^{+}, k} \mid \Xi_{t}=\xi_{t}\right]\right. \\
&\left.\quad+\mathbb{E}\left[\Sigma_{t^{+}, j} \cdot \Sigma_{t, k} \mid \Xi_{t}=\xi_{t}\right]+\mathbb{E}\left[\Sigma_{t^{+}, j} \cdot \Sigma_{t^{+}, k} \mid \Xi_{t}=\xi_{t}\right]\right) \\
& \\
& \quad \stackrel{(b)}{\leq}(1+\oslash)\left(\left(\epsilon_{j, k}+\oslash\right) \frac{\lambda}{2} \frac{\delta t}{h N}+\sqrt{\lambda} \frac{\delta t}{h N} 2 \pi(j+k)+\frac{\delta t}{h N}(2 \pi)^{2} j k\right) \\
& \quad \stackrel{(c)}{\leq}(1+\oslash)\left(\epsilon_{j, k}+\oslash\right) \frac{\lambda}{2} \frac{\delta t}{h N}(1+\oslash+\oslash) \leq_{\oslash}\left(\epsilon_{j, k}+\oslash\right) \frac{\lambda}{2} \frac{\delta t}{h N}
\end{aligned}
$$

with (a) a consequence of $-\infty \ll \mu_{k} \leq 0$ (Remark 4.13), (b) a consequence of Proposition 5.15 and (c) a consequences of $\lambda \approx \infty$ and standardness of $j, k \in \mathbb{Z}$.

\section{The main Theorem}

6.1 Remark We use-throughout section 6-the random variables $\widetilde{\Sigma}_{t}$ introduced in Definition 5.16 and the random coefficients of the series expansions with respect to $\left(g_{k}\right)_{k \in \mathcal{K}(h)}$ introduced in Definition 5.12.

6.2 Definition We define linear operators $\Phi_{-t}: \mathbb{R}^{\mathcal{H}} \rightarrow \mathbb{R}^{\mathcal{H}}$ by:

$$
g_{k} \mapsto \Phi_{-t}\left(g_{k}\right):=\frac{\prod_{u \in[0 \ldots T)}\left(1+\mu_{k} \delta u\right)}{\prod_{u \in[t \ldots T)}\left(1+\mu_{k} \delta u\right)(1+\lambda \delta u)} g_{k}=\frac{\prod_{u \in[0 \ldots t)}\left(1+\mu_{k} \delta u\right)}{\prod_{u \in[t \ldots T)}(1+\lambda \delta u)} g_{k}
$$

with $\left(g_{k}\right)_{k \in \mathcal{K}(h)}$ the eigenbasis of the discrete Laplace operator $\Delta_{h}$ introduced in Definition 4.7 and $\mu_{k}$ the respective eigenvalue of $\Delta_{h}$ that corresponds to $g_{k}$.

6.3 Definition Let $\Phi_{s}: \mathbb{R}^{\mathcal{H}} \rightarrow \mathbb{R}^{\mathcal{H}}$ be the linear operator given by

$$
g_{k} \mapsto \Phi_{s}\left(g_{k}\right):=\frac{\prod_{u \in(s \ldots T)}\left(1+\mu_{k} \delta u\right)(1+\lambda \delta u)}{\prod_{u \in[0 \ldots T)}\left(1+\mu_{k} \delta u\right)} g_{k}=\frac{\prod_{u \in(s \ldots T)}(1+\lambda \delta u)}{\prod_{u \in[0 \ldots s]}\left(1+\mu_{k} \delta u\right)} g_{k}
$$


and note that for $t>s$

$$
\left[\Phi_{-t} \circ \Phi_{s}\right]\left(g_{k}\right)=\prod_{u \in(s \ldots t)}\left(1+\mu_{k} \delta u\right)(1+\lambda \delta u) g_{k}
$$

Let further $\delta \Gamma_{s}:=\Phi_{s}\left(\widetilde{\Sigma}_{s}\right)$ and let $\Gamma_{t}:=\sum_{s \in[0 \ldots t)} \delta \Gamma_{s}$. Define further for $t \in$ $[\nu / \lambda \ldots T]$ random variables ${ }^{7}$

$$
\widetilde{\Gamma}_{t}:=\sum_{s \in[\nu / \lambda \ldots t)} \delta \Gamma_{s}=\Gamma_{t}-\Gamma_{\nu / \lambda}
$$

6.4 Proposition Suppose that Hypothesis 5.1 holds, let $[0 \ldots T]$ be an equally spaced near interval with spacing $\delta$ and let $\lambda^{2} \delta \approx 0$. Then for $s \in[0 \ldots T]$ we obtain

$$
\begin{gathered}
\Phi_{-s}\left(g_{k}\right)={ }_{\oslash} e^{\lambda(s-T)} e^{\mu_{k} s}(1+\oslash) g_{k} \\
\left\|\Phi_{s}\right\|_{2}:=\sup _{\xi \in \mathbb{S}\left(\mathbb{R}^{\mathcal{H}}\right)}\left\|\Phi_{s}(\xi)\right\|_{2} \leq_{\oslash} e^{\lambda T}(1+\oslash)
\end{gathered}
$$

Proof Equation (59) is a consequence of (56) and Proposition C.1, while (60) is a consequence of Definition 6.3, $\lambda \approx \infty$ and the fact that by Remark 4.13 we have $-\infty \ll \mu_{k} \leq 0$.

We display now the main theorem in the case that the number $\frac{1}{h} \in \mathbb{N}$ of positions occupied by particles in our dynamical system is standard.

6.5 Theorem Suppose that the Hypotheses 5.1 and 5.3 are fulfilled. ${ }^{8}$ Let $T, N, \lambda$ and $h$ be such that

$$
e^{2 \lambda T}={ }_{\oslash} 4 h N(1+\oslash) .
$$

Let the near interval $[0 \ldots T]$ be equally spaced and let the spacing $\delta$ be such that

$$
\lambda^{2} \delta \approx 0
$$

Let $\Gamma_{T}$ be the random variable introduced in Definition 6.3. Then

$$
\Gamma_{T} \sim_{\oslash} N\left(0, i d_{\mathcal{K}(h)}\right)
$$

Let $\nu \approx \infty$ be such that

$$
\nu / \lambda \in(0 \ldots T) \text { is infinitesimal and } T-\nu / \lambda \in(\nu / \lambda \ldots T)
$$

\footnotetext{
${ }^{7}$ We will specify $\nu \approx \infty$ in (64).

${ }^{8}$ Remember that $T \in(0, \infty)$ is limited by Hypothesis 5.1.
} 
and let $\Phi_{-t}$ be the linear operators introduced in Definition 6.2. Then for any $k \in \mathcal{K}(h)$

$$
\mathbb{P}\left(\max _{t \in[\nu / \lambda \ldots T]} \frac{\left|\Xi_{t, k}-\left(\Phi_{-t} \circ \Gamma_{T}\right)_{k}\right|^{2}}{\left|\left(\Phi_{-t} \circ \Gamma_{T}\right)_{k}\right|^{2}} \geq \frac{4 e^{-\nu}}{\Gamma_{T, k}^{2}} \wedge \Gamma_{T, k} \neq 0\right) \leq_{\oslash} e^{-\nu}(1+\oslash)
$$

$$
\forall t_{0} \in[\nu / \lambda \ldots T-\nu / \lambda] \quad \mathbb{P}\left(\max _{t \in\left[0 \ldots t_{0}\right]} \Xi_{t, k}^{2} \geq e^{-\lambda\left(T-t_{0}\right)}\right) \leq_{\oslash} e^{-\lambda\left(T-t_{0}\right)}(1+\oslash) \approx 0 .
$$

6.6 Remark Note that in the case that $T$ is appreciable (61) and (20) imply (62). Note that $\lambda \approx \infty$ and (62) imply $\lambda \delta \approx 0$. Further (56) implies that the trajectories $\left(y_{t}(\omega)\right)_{t \in[\nu / \lambda \ldots T]}:=\left(\left[\Phi_{-t} \circ \Gamma_{T}\right](\omega)\right)_{t \in[\nu / \lambda \ldots T]}$ fulfill $y_{t+\delta t}=L y_{t}$ with $L$ the linear transformation given by $L g_{k}=\left(1+\mu_{k} \delta t\right)(1+\lambda \delta t) g_{k}$.

6.7 Corollary Under the hypotheses of Theorem $6.5(\nu \approx \infty$ etc.) we obtain for any standard $\varepsilon>0$ that

$$
\mathbb{P}\left(\max _{t \in[\nu / \lambda \ldots T]} \frac{\left|\Xi_{t, k}-\left(\Phi_{-t} \circ \Gamma_{T}\right)_{k}\right|^{2}}{\left|\left(\Phi_{-t} \circ \Gamma_{T}\right)_{k}\right|^{2}} \geq e^{-\nu(1-\varepsilon)} \mid \Gamma_{T, k}^{2} \geq 4 e^{-\nu \varepsilon}\right) \leq_{\oslash} e^{-\nu}(1+\oslash) .
$$

Derivation of Corollary 6.7 from Theorem 6.5 We calculate

$$
\begin{aligned}
& \mathbb{P}\left(\max _{t \in[\nu / \lambda \ldots T]} \frac{\left|\Xi_{t, k}-\left(\Phi_{-t} \circ \Gamma_{T}\right)_{k}\right|^{2}}{\left|\left(\Phi_{-t} \circ \Gamma_{T}\right)_{k}\right|^{2}} \geq e^{-\nu(1-\varepsilon)} \mid \Gamma_{T, k}^{2} \geq 4 e^{-\nu \varepsilon}\right) \cdot \mathbb{P}\left(\Gamma_{T, k}^{2} \geq 4 e^{-\nu \varepsilon}\right) \\
& \quad \leq \mathbb{P}\left(\max _{t \in[\nu / \lambda \ldots T]} \frac{\left|\Xi_{t, k}-\left(\Phi_{-t} \circ \Gamma_{T}\right)_{k}\right|^{2}}{\left|\left(\Phi_{-t} \circ \Gamma_{T}\right)_{k}\right|^{2}} \geq \frac{4 e^{-\nu}}{\Gamma_{T, k}^{2}} \mid \Gamma_{T, k}^{2} \geq 4 e^{-\nu \varepsilon}\right) \cdot \mathbb{P}\left(\Gamma_{T, k}^{2} \geq 4 e^{-\nu \varepsilon}\right) \\
& \quad \leq \mathbb{P}\left(\max _{t \in[\nu / \lambda \ldots T]} \frac{\left|\Xi_{t, k}-\left(\Phi_{-t} \circ \Gamma_{T}\right)_{k}\right|^{2}}{\left|\left(\Phi_{-t} \circ \Gamma_{T}\right)_{k}\right|^{2}} \geq \frac{4 e^{-\nu}}{\Gamma_{T, k}^{2}} \wedge \Gamma_{T, k} \neq 0\right) \stackrel{(65)}{\leq} e_{\oslash} e^{-\nu}(1+\oslash) .
\end{aligned}
$$

From (63), Remark 4.20 and $\nu \approx \infty$ we obtain that

$$
\mathbb{P}\left(\Gamma_{T, k}^{2} \geq 4 e^{-\nu \varepsilon}\right)={ }_{\oslash} 1+\oslash
$$

and from (68) and (69) we obtain that (67) holds.

Derivation of Theorem 3.1 from Theorem 6.5 and Corollary 6.7 Formula (10) is an immediate consequence of (66) (consider the case $t_{0}=T-\nu / \lambda$ ), while (9) holds 
since for all standard $\varepsilon>0$

$$
\begin{aligned}
& \mathbb{P}\left(\Gamma_{T} \neq 0 \wedge \max _{t \in[\nu / \lambda \ldots T]} \frac{\left\|\Xi_{t}(\omega)-\left[\Phi_{-t} \circ \Gamma_{T}\right](\omega)\right\|_{2}^{2}}{\left\|\Phi_{-t} \circ \Gamma_{T}(\omega)\right\|_{2}^{2}} \geq e^{-\nu(1-\varepsilon)}\right) \\
& \leq \sum_{k \in \mathcal{K}(h)} \mathbb{P}\left(\max _{t \in[\nu / \lambda \ldots T]} \frac{\left|\Xi_{t, k}-\left(\Phi_{-t} \circ \Gamma_{T}\right)_{k}\right|^{2}}{\left|\left(\Phi_{-t} \circ \Gamma_{T}\right)_{k}\right|^{2}} \geq e^{-\nu(1-\varepsilon)} \mid \Gamma_{T, k}^{2} \geq 4 e^{-\nu \varepsilon}\right) \\
& \quad+\sum_{k \in \mathcal{K}(h)} \mathbb{P}\left(\Gamma_{T, k}^{2} \leq 4 e^{-\nu \varepsilon}\right) \\
& \quad \stackrel{(a)}{\leq} e^{-\nu}(1+\oslash) h^{-1}+\oslash h^{-1} \stackrel{(b)}{\approx} 0
\end{aligned}
$$

with (a) a consequence of (69) and (67) and (b) a consequence of $\nu \approx \infty$ and the standardness of $h$.

6.8 Remark By the axiom of idealization Theorem 6.5 extends to the hyperfinite situation:

6.9 Theorem There exists a $\chi \in \overline{\mathbb{N}}$ such that Theorem 6.5 still holds if the hypothesis (19) is replaced by

$$
\mathbb{N} \ni \frac{1}{h} \leq \chi
$$

Derivation of Theorem 6.9 from Theorem 6.5 By Remark 4.24 formula (63) says that for any standard $\varepsilon \in(0, \infty)$ and for any standard $f \in \mathcal{C}_{b}(\mathbb{R})$ we have that:

$$
\left(\forall \psi^{*} \in \mathbb{S}^{*}\left(\mathbb{R}^{\mathcal{K}(h)}\right)\right) \quad\left(\left|\mathbb{E}\left[f \circ \psi^{*}\left(\Gamma_{T}\right)\right]-\int_{y \in \mathbb{R}} f(y) \frac{\exp \left(-y^{2} / 2\right)}{\sqrt{2 \pi}} d y\right|<\varepsilon\right)
$$

Since Theorem 6.5 holds for any $h \in\{1 / n \mid n \in \underline{\mathbb{N}}\}$ we obtain that (71) holds for any $(h, f, \varepsilon)$ with $(h, f, \varepsilon) \in\{1 / n \mid \chi \geq n \in \mathbb{N}\} \times \mathcal{C} \times \mathcal{E}$ with $\mathcal{C} \times \mathcal{E}$ an arbitrary standard finite subset of $\mathcal{C}_{b}(\mathbb{R}) \times(0, \infty)$ and $\chi \in \mathbb{N}$ standard. By an application of the idealization axiom of IST we obtain that (71) holds for any $(h, f, \varepsilon) \in\{1 / n \mid \tilde{\chi} \geq n \in \mathbb{N}\} \times \mathcal{C} \times \mathcal{E}$ with $\tilde{\chi} \in \overline{\mathbb{N}}$ and $\mathcal{C} \times \mathcal{E}$ a finite set containing all standard elements of $\mathcal{C}_{b}(\mathbb{R}) \times(0, \infty)$. I.e., (71) holds for any standard $\varepsilon \in(0, \infty)$, for any standard $f \in \mathcal{C}_{b}(\mathbb{R})$ and any $h \in\{1 / n \mid \tilde{\chi} \geq n \in \mathbb{N}\}$, and thus (63) holds for any $h$ that fulfills (70). That there exists a $\hat{\chi} \in \overline{\mathbb{N}}$ such that (65) and (66) hold for any $h$ that fulfills $\mathbb{N} \ni 1 / h \leq \hat{\chi}$ is obtained by application of idealization in an analogous manner. To complete the proof of the theorem simply let $\chi:=\min (\widetilde{\chi}, \widehat{\chi})$.

6.10 Remark Before we prove Theorem 6.5 we prove Proposition 6.11 that expresses the system's dynamics with respect to $\Gamma_{t}$, Proposition 6.12 that provides some information concerning $\delta \Gamma_{s}$, Lemma 6.14 that gives a formula for summing the variances of 
the $\delta \Gamma_{s}$ and Lemma 6.15 that proves (65) and prepares for the final steps in the proof of Theorem 6.5.

6.11 Proposition Suppose that the linear operators $\Phi_{-t}$ are given by Definition 6.2 and the random variables $\delta \Gamma_{t}$ and $\Gamma_{t}$ by Definition 6.3. Then

$$
\Xi_{t, k}=\sum_{s \in[0 \ldots t)}\left(\prod_{u \in(s \ldots t)}(1+\lambda \delta u)\left(1+\mu_{k} \delta u\right)\right) \widetilde{\Sigma}_{s, k}
$$

or equivalently

$$
\Xi_{t}=\sum_{s \in[0 \ldots t)}\left[\Phi_{-t} \circ \Phi_{s}\right]\left(\widetilde{\Sigma}_{s}\right)=\sum_{s \in[0 \ldots t)} \Phi_{-t}\left(\delta \Gamma_{s}\right)=\Phi_{-t}\left(\Gamma_{t}\right) .
$$

Proof By Definition 5.16 the recursion (44) becomes

$$
\Xi_{u+\delta u, k}=\left(1+\mu_{k} \delta u\right)(1+\lambda \delta u) \Xi_{u, k}+\widetilde{\Sigma}_{u, k} .
$$

From $\Xi_{0}=0$ and (74) we obtain by recursion that the coordinate wise system's dynamics is given by (72), while equation (73) is just a reformulation of (72) using the Definition 6.3 and especially (57).

6.12 Proposition Suppose that the hypotheses of Theorem 6.5 are fulfilled, and that $\delta \Gamma_{s}, \Gamma_{s}$ and $\Phi_{s}$ are given by Definition 6.3. Let $\psi^{*} \in \mathbb{S}^{*}\left(\mathbb{R}^{\mathcal{H}}\right)$ be arbitrarily given and let $\psi_{k}:=\psi^{*}\left(g_{k}\right)$. Then (denoting the inverse of $\Phi_{-s}$ by $\Phi_{-s}^{-1}$ )

$$
\begin{gathered}
\forall \omega \in \Omega \quad\left\|\delta \Gamma_{s}(\omega)\right\|_{2} \approx 0 \\
\mathbb{E}\left[\psi^{*} \circ \delta \Gamma_{s} \mid \Gamma_{s}\right]=\mathbb{E}\left[\psi^{*} \circ \Phi_{s} \circ \widetilde{\Sigma}_{s} \mid \Phi_{-s}^{-1} \circ \Xi_{s}\right]=0 \\
\mathbb{E}\left[\left(\psi^{*}\right)^{2} \circ \delta \Gamma_{s} \mid \Gamma_{s}\right]={ }_{\diamond}(1+\oslash) \frac{\lambda}{2} \frac{\delta}{h N} \sum_{k \in \mathcal{K}(h)} \frac{(1+\lambda \delta)^{2(T-s) / \delta}}{\left(1+\mu_{k} \delta\right)^{2 s / \delta}} \psi_{k}^{2} \approx 0 .
\end{gathered}
$$

Proof We conclude (75) from

$$
\begin{aligned}
\left\|\delta \Gamma_{s}(\omega)\right\|_{2}=\left\|\Phi_{s} \circ \widetilde{\Sigma}_{s}(\omega)\right\|_{2} & \leq\left\|\Phi_{s}\right\|_{2}\left\|\widetilde{\Sigma}_{s}(\omega)\right\|_{2} \\
& \stackrel{(a)}{\leq} e^{\lambda T} \frac{8}{h N}(1+\oslash) \stackrel{(b)}{\leq} 32 e^{-\lambda T}(1+\oslash) \approx 0
\end{aligned}
$$

with (a) a consequence of (60) and (52), and (b) a consequence of (61). Further (76) is a consequence of (73), Definition 6.3, (53) and the linearity of $\psi^{*} \circ \Phi_{s}$. Finally we 
obtain (77) by the following calculation:

$$
\begin{aligned}
& \mathbb{E}\left(\left(\psi^{*}\right)^{2} \circ \delta \Gamma_{s} \mid \Gamma_{s}\right)=\mathbb{E}\left[\left(\psi^{*} \circ \Phi_{s} \circ \widetilde{\Sigma}_{s}\right)^{2} \mid \Phi_{-s}^{-1} \circ \Xi_{s}\right] \\
& =\mathbb{E}\left[\left(\psi^{*} \circ \Phi_{s} \circ \sum_{k \in \mathcal{K}(h)} \widetilde{\Sigma}_{s, k} g_{k}\right)^{2} \mid \Phi_{-s}^{-1} \circ \Xi_{s}\right] \\
& \stackrel{(a)}{=} \mathbb{E}\left[\left(\sum_{k \in \mathcal{K}(h)} \frac{\prod_{u \in(s \ldots T)}(1+\lambda \delta)}{\prod_{u \in[0 \ldots s]}\left(1+\mu_{k} \delta\right)} \psi_{k} \widetilde{\Sigma}_{s, k}\right)^{2} \mid \Phi_{-s}^{-1} \circ \Xi_{s}\right] \\
& =\oslash \sum_{j, k \in \mathcal{K}(h)} \frac{(1+\lambda \delta)^{2(T-s) / \delta}(1+\oslash)}{\left(1+\mu_{j} \delta\right)^{s / \delta}\left(1+\mu_{k} \delta\right)^{s / \delta}} \psi_{j} \psi_{k} \mathbb{E}\left[\widetilde{\Sigma}_{s, j} \cdot \widetilde{\Sigma}_{s, k} \mid \Phi_{-s}^{-1} \circ \Xi_{s}\right] \\
& \stackrel{(b)}{=}(1+\oslash) \sum_{k \in \mathcal{K}(h)} \frac{(1+\lambda \delta)^{2(T-s) / \delta}}{\left(1+\mu_{k} \delta\right)^{2 s / \delta}} \psi_{k}^{2} \frac{\lambda}{2} \frac{\delta}{h N} \leq_{\oslash} \frac{e^{2 \lambda T}}{4 h N} 2 \lambda \delta(1+\oslash) \stackrel{(c)}{\approx} 0 .
\end{aligned}
$$

Equality (a) follows since $\psi_{k}=\psi^{*}\left(g_{k}\right)$. Equality (b) follows from (54) since $\mathcal{K}(h)$ is a standard finite set, while (c) follows from (61) and $\lambda \delta \approx 0$ (Remark 6.6).

6.13 Remark Note that for any $k \in \mathcal{K}(h)$ the stochastic processes $\left(\Gamma_{t, k}\right)_{t \in[0 \ldots T]}$ and $\left(\widetilde{\Gamma}_{t, k}\right)_{t \in[\nu / \lambda \ldots T]}$ are by (76) with $\psi^{*}(\gamma)=\left\langle\gamma \mid g_{k}\right\rangle$ and Definition 6.3 martingales.

6.14 Lemma Suppose that $\lambda \approx \infty, \nu \approx \infty, 0 \approx \nu / \lambda<T \ll \infty, \lambda^{2} \delta \approx 0$, $-\infty \ll \mu_{k} \leq 0$ and $e^{2 \lambda T}=\oslash 4 h N(1+\oslash)$. Then

$$
\frac{\lambda}{2} \frac{\delta}{h N} \sum_{s \in[0 \ldots \nu / \lambda)} \frac{(1+\lambda \delta)^{2(T-s) / \delta}}{\left(1+\mu_{k} \delta\right)^{2 s / \delta}}={ }_{\oslash}(1+\oslash) \frac{e^{2 \lambda T}}{4 h N}={ }_{\oslash}(1+\oslash)
$$

Proof Equation (78) is proved by the following calculation

$$
\begin{aligned}
& \frac{\lambda}{2} \frac{\delta}{h N} \sum_{s \in[0 \ldots \nu / \lambda)} \frac{(1+\lambda \delta)^{2(T-s) / \delta}}{\left(1+\mu_{k} \delta\right)^{2 s / \delta}} \\
& =\frac{\lambda}{2} \frac{\delta}{h N} \frac{1}{\left(1+\mu_{k} \delta\right)^{2 T / \delta}} \sum_{s \in[0 \ldots \nu / \lambda)}\left((1+\lambda \delta)\left(1+\mu_{k} \delta\right)\right)^{2(T-s) / \delta} \\
& \stackrel{(a)}{=} \frac{\lambda}{2} \frac{\delta}{h N} \frac{1}{\left(1+\mu_{k} \delta\right)^{2 T / \delta}} \frac{\left((1+\lambda \delta)\left(1+\mu_{k} \delta\right)\right)^{2 T / \delta+2}-\left((1+\lambda \delta)\left(1+\mu_{k} \delta\right)\right)^{2\left(T-\frac{\nu}{\lambda}\right) / \delta+2}}{\left((1+\lambda \delta)\left(1+\mu_{k} \delta\right)\right)^{2}-1} \\
& =\frac{\lambda}{2} \frac{\delta}{h N}(1+\lambda \delta)^{2 T / \delta} \frac{\left((1+\lambda \delta)\left(1+\mu_{k} \delta\right)\right)^{2}-\left((1+\lambda \delta)\left(1+\mu_{k} \delta\right)\right)^{-2 \frac{\nu}{\lambda} / \delta+2}}{\left((1+\lambda \delta)\left(1+\mu_{k} \delta\right)\right)^{2}-1} \\
& \stackrel{(b)}{=}{ }_{\oslash}(1+\oslash) \frac{\lambda}{2} \frac{\delta}{h N} e^{2 \lambda T} \cdot \frac{(1+\oslash)-(1+\oslash) e^{-2\left(\lambda+\mu_{k}\right) \frac{\nu}{\lambda}}}{2\left(\lambda+\mu_{k}+\oslash\right) \delta}={ }_{\oslash}(1+\oslash) \frac{e^{2 \lambda T}}{4 h N} \stackrel{(c)}{=}_{\oslash}(1+\oslash) .
\end{aligned}
$$


Here (a) follows from an application of the formula $\sum_{j=m}^{n} x^{j}=\frac{x^{n+1}-x^{m}}{x-1}$ for summing finite geometric series, while $(b)$ follows by application of Proposition C.1 in the cases $(\kappa, t)=(\lambda, T),(\kappa, t)=(\lambda, \nu / \lambda)$ and $(\kappa, t)=\left(\mu_{k}, \nu / \lambda\right)$. The equality $(c)$ finally follows from $\nu \approx \infty, \lambda /\left(\lambda+\mu_{k}+\oslash\right)={ }_{\varnothing}(1+\oslash)$ and $(61)$.

6.15 Lemma Suppose that the hypotheses of Theorem 6.5 are fulfilled, that linear operators $\Phi_{-t}$ are given by Definition 6.2 and random variables $\delta \Gamma_{t}, \Gamma_{t}$ and $\widetilde{\Gamma}_{t}$ by Definition 6.3. Then we obtain for $t \in[\nu / \lambda \ldots T], k \in \mathcal{K}(h), \psi^{*} \in \mathbb{S}^{*}\left(\mathbb{R}^{\mathcal{H}}\right)$ and $\psi_{k}:=\psi^{*}\left(g_{k}\right)$ that:

The random variable $\Xi_{t}$ can be decomposed by linearity of $\Phi_{-t}$ as

$$
\Xi_{t}=\Phi_{-t}\left(\Gamma_{\frac{\nu}{\lambda}}+\widetilde{\Gamma}_{t}\right)=\left(\Phi_{-t}\left(\Gamma_{\frac{\nu}{\lambda}}\right)+\Phi_{-t}\left(\widetilde{\Gamma}_{t}\right)\right)
$$

The variances $\mathbb{E}\left[\left(\psi^{*}\right)^{2} \circ \widetilde{\Gamma}_{t_{0}}\right]$ and $\mathbb{E}\left[\left(\psi^{*}\right)^{2} \circ \Gamma_{t_{0}}\right]$ can be estimated by

$$
\begin{gathered}
\left(\forall t_{0} \in[\nu / \lambda \ldots T]\right)\left(\mathbb{E}\left[\left(\psi^{*}\right)^{2} \circ \widetilde{\Gamma}_{t_{0}}\right] \leq_{\oslash} e^{-2 \nu}(1+\oslash)\right) \\
\left(\forall t_{0} \in[\nu / \lambda \ldots T]\right)\left(\mathbb{E}\left[\left(\psi^{*}\right)^{2} \circ \Gamma_{t_{0}}\right]=_{\oslash}(1+\oslash)\right)
\end{gathered}
$$

The distributions of $\psi^{*} \circ \Gamma_{\frac{\nu}{\lambda}}$ and $\psi^{*} \circ \widetilde{\Gamma}_{T}$ fulfill

$$
\begin{gathered}
\psi^{*} \circ \widetilde{\Gamma}_{T} \sim_{\oslash} N\left(0, e^{-2 \nu}(1+\oslash)\right) \\
\psi^{*} \circ \Gamma_{\frac{\nu}{\lambda}} \sim_{\oslash} N(0,(1+\oslash))
\end{gathered}
$$

The maximum of $\widetilde{\Gamma}_{t, k}^{2}$ is bounded by

$$
\mathbb{P}\left(\max _{t \in[\nu / \lambda \ldots T]} \widetilde{\Gamma}_{t, k}^{2} \geq e^{-\nu}\right) \leq e^{\nu} \mathbb{E}\left[\widetilde{\Gamma}_{T, k}^{2}\right] \leq_{\oslash} e^{-\nu}(1+\oslash)
$$

and (65) holds.

Proof Equation (79) that describes the system's dynamics with respect to the random variable $\Gamma_{\frac{\nu}{\lambda}}$ and the stochastic process $\left(\widetilde{\Gamma}_{t}\right)_{t \in[\nu / \lambda \ldots T]}$ follows from (73) and (58). Next we show that the distribution-properties of $\Gamma$ and $\widetilde{\Gamma}$ displayed in (82) and (83) are consequences of (80), (81) and Proposition 6.12. (We just prove $(81) \Rightarrow(83)$ since $(80) \Rightarrow(82)$ follows completely analogous.) To this end we apply Theorem A.7 to the Markov process $\left(\Gamma_{t}\right)_{t \in[0 \ldots \nu / \lambda]}$ introduced in Definition 6.3. We notice that $\delta \Gamma_{t}=\sum_{\{\theta \mid(t, \theta) \in \mathbb{G}\}}^{\circ} \delta \Gamma_{t, \theta}$ where $\sum^{\circ}$ denotes disjoint union, ${ }^{9} \delta \Gamma_{t}$ denotes the increments of $\left(\Gamma_{t}\right)_{t \in[0 \ldots \nu / \lambda]}$ introduced in Definition 6.3, and $\mathbb{G}, \delta \Gamma_{t, \theta}$ denote the objects introduced

\footnotetext{
${ }^{9}$ We identify functions with their graphs.
} 
in Theorem A.7. Since $[0 \ldots \nu / \lambda)$ is (by (20) and since $\nu \approx \infty$ ) hyperfinite the conclusions of Proposition 6.12 imply the hypotheses of Theorem A.7 with

$$
\sigma_{t}^{2}=\frac{\lambda}{2} \frac{\delta}{h N} \sum_{k \in \mathcal{K}(h)} \frac{(1+\lambda \delta)^{2(T-s) / \delta}}{\left(1+\mu_{k} \delta\right)^{2 s / \delta}} \psi_{k}^{2} .
$$

Theorem A.7 therefore applies and-making use of (81) with $t_{0}=\nu / \lambda$-proves (83), i.e., $(81) \Rightarrow(83)$ and $(80) \Rightarrow(82)$ hold.

The equations (80) and (81) are simple consequences of (76) and

$$
\begin{aligned}
\sum_{s \in[0 \ldots \nu / \lambda)} \mathbb{E}\left[\left(\psi^{*}\right)^{2} \circ \delta \Gamma_{s} \mid \Gamma_{s}\right] & ={ }_{\oslash}(1+\oslash) \\
\sum_{s \in[\nu / \lambda \ldots T)} \mathbb{E}\left[\left(\psi^{*}\right)^{2} \circ \delta \Gamma_{s} \mid \Gamma_{s}\right] & ={ }_{\oslash} e^{-2 \nu}(1+\oslash) .
\end{aligned}
$$

We just prove (85), since the proof of (86) is analogous. To prove (85), however, it is by (77) and $\sum_{k \in \mathcal{K}(h)} \psi_{k}^{2}=1$ clearly sufficient to prove

$$
\frac{\lambda}{2} \frac{\delta}{h N} \sum_{s \in[0 \ldots \nu / \lambda)} \frac{(1+\lambda \delta)^{2(T-s) / \delta}}{\left(1+\mu_{k} \delta\right)^{2 s / \delta}}={ }_{\oslash}(1+\oslash),
$$

i.e., to apply Lemma 6.14. Consequently we established (85), (86) and thus (80), (81) and further (82), (83).

Inequality (84) is a consequence of (80) with $\psi^{*}(\gamma)=\left\langle\gamma \mid g_{k}\right\rangle$, Remark 6.13 and the Doob inequality stated as Proposition C.3.

Thus it remains to prove (65). Under the hypothesis $\Gamma_{T, k} \neq 0$ we calculate:

$$
\begin{gathered}
\max _{t \in[\nu / \lambda \ldots T]} \frac{\left|\Xi_{t, k}-\left(\Phi_{-t} \circ \Gamma_{T}\right)_{k}\right|^{2}}{\left|\left(\Phi_{-t} \circ \Gamma_{T}\right)_{k}\right|^{2}} \stackrel{(a)}{=} \max _{t \in[\nu / \lambda \ldots T]} \frac{\left|\left(\Phi_{-t}\left(\Gamma_{t}\right)\right)_{k}-\left(\Phi_{-t}\left(\Gamma_{T}\right)\right)_{k}\right|^{2}}{\left|\Phi_{-t}\left(\Gamma_{T}\right)_{k}\right|^{2}} \\
\stackrel{(b)}{=} \max _{t \in[\nu / \lambda \ldots T]} \frac{\left|\Gamma_{t, k}-\Gamma_{T, k}\right|^{2}}{\left|\Gamma_{T, k}\right|^{2}} \stackrel{(c)}{\leq} \max _{t \in[\nu / \lambda \ldots T]} \frac{\left(\left|\widetilde{\Gamma}_{t, k}\right|+\left|\widetilde{\Gamma}_{T, k}\right|\right)^{2}}{\left|\Gamma_{T, k}\right|^{2}} \leq \max _{t \in[\nu / \lambda \ldots T]} \frac{4 \widetilde{\Gamma}_{t, k}^{2}}{\Gamma_{T, k}^{2}}
\end{gathered}
$$

with (a), (b) and (c) consequences of (73), (56) and (58), respectively. We conclude that

$$
\begin{array}{r}
\mathbb{P}\left(\max _{t \in[\nu / \lambda \ldots T]} \frac{\left|\Xi_{t, k}-\left(\Phi_{-t} \circ \Gamma_{T}\right)_{k}\right|^{2}}{\left|\left(\Phi_{-t} \circ \Gamma_{T}\right)_{k}\right|^{2}} \geq \frac{4 e^{-\nu}}{\Gamma_{T, k}^{2}} \wedge \Gamma_{T, k} \neq 0\right) \\
\stackrel{(a)}{\leq} \mathbb{P}\left(\max _{t \in[\nu / \lambda \ldots T)} 4 \widetilde{\Gamma}_{t, k}^{2} \geq 4 e^{-\nu}\right) \stackrel{(b)}{\leq} e_{\oslash} e^{-\nu}(1+\oslash)
\end{array}
$$

with (a) a consequence of (87) and (b) a consequence of (84), i.e., we conclude that (65) holds. 
Proof of Theorem 6.5 From (83) and (82) we obtain

$$
\forall \psi^{*} \in \mathbb{S}^{*}\left(\mathbb{R}^{\mathcal{H}}\right) \quad \psi^{*} \circ \Gamma_{T}=\psi^{*} \circ \Gamma_{\nu / \lambda}+\psi^{*} \circ \widetilde{\Gamma}_{T} \sim_{\oslash} N(0,(1+\oslash)) .
$$

From (88) we obtain by Definition 4.22 that $\Gamma_{T} \sim_{\oslash} N\left(0, i d_{\mathcal{K}(h)}\right)$, i.e., (63) has been proved. Since (65) has already been proved (Lemma 6.15) it remains to show (66). This is done by calculating for $t_{0} \in[\nu / \lambda \ldots T]$

$$
\begin{aligned}
& \mathbb{P}\left(\max _{t \in\left[0 \ldots t_{0}\right]} \Xi_{t, k}^{2} \geq e^{-\lambda\left(T-t_{0}\right)}\right) \stackrel{(a)}{=} \mathbb{P}\left(\max _{t \in\left[0 \ldots t_{0}\right]}\left(\Phi_{-t} \circ \Gamma_{t}\right)_{k}^{2} \geq e^{-\lambda\left(T-t_{0}\right)}\right) \\
& \quad \stackrel{(b)}{\leq}_{\oslash} \mathbb{P}\left(\max _{t \in\left[0 \ldots t_{0}\right]} \Gamma_{t, k}^{2} \geq_{\oslash} e^{\lambda\left(T-t_{0}\right)} e^{-2 \mu_{k} t_{0}}(1+\oslash)\right) \\
& \quad \stackrel{(c)}{\leq} \mathbb{P}\left(\max _{t \in\left[0 \ldots t_{0}\right]} \Gamma_{t, k}^{2} \geq_{\oslash} e^{\lambda\left(T-t_{0}\right)}(1+\oslash)\right) \stackrel{(d)}{\leq} e_{\oslash} e^{-\lambda\left(T-t_{0}\right)}(1+\oslash)^{-1} \mathbb{E}\left[\Gamma_{t_{0}, k}^{2}\right] \\
& \quad \stackrel{(e)}{\leq} e^{-\lambda\left(T-t_{0}\right)}(1+\oslash)
\end{aligned}
$$

with (a) a consequence of (73), (b) a consequence of (59), (c) a consequence of the fact that $\mu_{k} \leq 0$ (Remark 4.13), (d) a consequence of Remark 6.13 and Proposition C.3, and (e) a consequence of (81).

\section{Reformulation as a standard limit theorem}

7.1 Remark In this section we formulate a limit result (Theorem 7.9). This limit theorem is still formulated within the realm of finite probability spaces. Its corollary 7.11 makes however use of random variables that are $N(0, i d)$ distributed in the usual ZFC based sense (not in our IST based approximate sense). Such random variables can not be defined on a finite or countable probability space. For general measure theoretic probability theory adequate for dealing with random variables on uncountable spaces we refer the reader to Dudley [9].

7.2 Remark We reformulate parts of Theorem 6.5 as a limit theorem in standard mathematical terms. To do this we have to consider sequences of interacting particle systems instead of a single system. We therefore replace the mathematical objects $N, \lambda$, $\delta, \Omega,[0 \ldots T], \mathbb{X}, \Xi, \widetilde{\Sigma} \Gamma, \widetilde{\Gamma}$ and $\Phi$ introduced in the sections 5 and 6 by sequences $\left(N^{\beta}\right)_{\beta \in \mathbb{N}},\left(\lambda^{\beta}\right)_{\beta \in \mathbb{N}},\left(\delta^{\beta}\right)_{\beta \in \mathbb{N}},\left(\Omega^{\beta}\right)_{\beta \in \mathbb{N}},\left(\left[0 \ldots T^{\beta}\right]\right)_{\beta \in \mathbb{N}},\left(\mathbb{X}^{\beta}\right)_{\beta \in \mathbb{N}},\left(\Xi^{\beta}\right)_{\beta \in \mathbb{N}},\left(\widetilde{\Sigma}^{\beta}\right)_{\beta \in \mathbb{N}}$, $\left(\Gamma^{\beta}\right)_{\beta \in \mathbb{N}}$, $\left(\widetilde{\Gamma}^{\beta}\right)_{\beta \in \mathbb{N}}$ and $\left(\Phi^{\beta}\right)_{\beta \in \mathbb{N}}$. Note that $\left(\left[0 \ldots T^{\beta}\right]\right)_{\beta \in \mathbb{N}}$ denotes a sequence of finite sets, whose terms are not necessarily near intervals, i.e., $\left[0 \ldots T^{\beta}\right]$ denotes in this section a finite set that is not necessarily a near interval. 
7.3 Hypothesis Let $\left(N^{\beta}\right)_{\beta \in \mathbb{N}} \in \mathbb{N}^{\mathbb{N}},\left(\lambda^{\beta}\right)_{\beta \in \mathbb{N}} \in(0, \infty)^{\mathbb{N}}$ be sequences such that $\lim _{\beta \in \mathbb{N}} N^{\beta}=\infty, \lim _{\beta \in \mathbb{N}} \lambda^{\beta}=\infty$. Let $\left(\left[0 \ldots T^{\beta}\right]\right)_{\beta \in \mathbb{N}}$ be a sequence of equally spaced finite sets $\left[0 \ldots T^{\beta}\right]$ with spacings $\delta^{\beta}$ such that $\left\{0, T^{\beta}\right\} \subseteq\left[0 \ldots T^{\beta}\right] \subset\left[0, T^{\beta}\right]$ and $\lim \sup _{\beta \in \mathbb{N}} T^{\beta} \in[0, \infty)$. Let $h \in\left\{\frac{1}{n} \mid n \in \mathbb{N}\right\}$ be independent of $\beta$ and suppose

$$
\lim _{\beta \rightarrow \infty} \delta^{\beta} \lambda^{\beta} h N^{\beta}=0, \quad \lim _{\beta \rightarrow \infty}\left(\lambda^{\beta}\right)^{2} \delta^{\beta}=0 \quad \text { and } \quad \lim _{\beta \rightarrow \infty} \frac{e^{2 \lambda^{\beta} T^{\beta}}}{4 h N^{\beta}}=1 .
$$

7.4 Hypothesis Let $\left(N^{\beta}\right)_{\beta \in \mathbb{N}},\left(\lambda^{\beta}\right)_{\beta \in \mathbb{N}},\left(\Xi^{\beta}\right)_{\beta \in \mathbb{N}}$ etc. be sequences, such that for any $\beta \in \mathbb{N}$ Hypothesis 5.3 holds with $N, \lambda, \Xi$ etc. replaced by $N^{\beta}, \lambda^{\beta}, \Xi^{\beta}$ etc. (with $\mathbb{X}^{\beta}$, $\mathcal{H}, \mathcal{K}(h)$ provided in analogy with Hypothesis 5.1).

7.5 Definition Define a sequence $\left(\Phi_{-}^{\beta}\right)_{\beta \in \mathbb{N}}$ of parametric families $\Phi_{-}^{\beta}=\left(\Phi_{-t}^{\beta}\right)_{t \in\left[0 \ldots T^{\beta}\right]}$ of linear operators $\Phi_{-t}^{\beta}: \mathbb{R}^{\mathcal{H}^{\beta}} \rightarrow \mathbb{R}^{\mathcal{H}^{\beta}}$ by:

$$
g_{k} \mapsto \Phi_{-t}^{\beta}\left(g_{k}\right):=\frac{\prod_{u \in[0 \ldots t)^{\beta}}\left(1+\mu_{k} \delta u\right) g_{k}}{\prod_{u \in\left[t \ldots T^{\beta}\right)}\left(1+\lambda^{\beta} \delta u\right)}
$$

with $[0 \ldots t)^{\beta}:=\left\{u \in\left[0 \ldots T^{\beta}\right) \mid u<t\right\},\left[t \ldots T^{\beta}\right):=\left\{u \in\left[0 \ldots T^{\beta}\right) \mid t \leq\right.$ $\left.u<T^{\beta}\right\},\left(g_{k}\right)_{k \in \mathcal{K}(h)^{\beta}}$ the eigenbasis of the discrete Laplace operator $\Delta_{h}$ introduced in Definition 4.7 and $\mu_{k}$ the respective eigenvalue of $\Delta_{h}$ that corresponds to $g_{k}$.

7.6 Definition Define the sequence $\left(\Gamma_{T^{\beta}}^{\beta}\right)_{\beta \in \mathbb{N}}$ of random variables $\Gamma_{T^{\beta}}^{\beta}$ in analogy with the definitions given in the sections 5 and 6 starting with $\Xi^{\beta}$ instead of $\Xi$ and replacing objects like $\widetilde{\Sigma}, \Phi$ etc. in the consecutive Definitions 5.6, 5.12, 5.16 and 6.3 by consecutively defined objects $\widetilde{\Sigma}^{\beta}$, $\Phi^{\beta}$ etc.

7.7 Hypothesis Let $\left(N^{\beta}\right)_{\beta \in \mathbb{N}} \in \mathbb{N}^{\mathbb{N}}$ and $\left(\lambda^{\beta}\right)_{\beta \in \mathbb{N}} \in(0, \infty)^{\mathbb{N}}$ be sequences such that for all $\beta \in \overline{\mathbb{N}}$ we have $N^{\beta} \in \overline{\mathbb{N}}, \lambda^{\beta} \approx \infty$. Suppose that $\left(\left[0 \ldots T^{\beta}\right]\right)_{\beta \in \mathbb{N}}$ is a sequence of equally spaced finite sets $\left[0 \ldots T^{\beta}\right]$ with spacings $\delta^{\beta}$ such that $\forall \beta \in \overline{\mathbb{N}}$ the set $\left[0 \ldots T^{\beta}\right]$ is a near interval and $0<T^{\beta} \ll \infty$. Let $h \in\left\{\frac{1}{n} \mid n \in \mathbb{N}\right\}$ be independent of $\beta$ and suppose that:

$$
(\forall \beta \in \overline{\mathbb{N}}) \quad\left(\delta^{\beta} \lambda^{\beta} h N^{\beta} \approx 0,\left(\lambda^{\beta}\right)^{2} \delta^{\beta} \approx 0 \text { and } e^{2 \lambda^{\beta} T^{\beta}}={ }_{\varnothing} 4 h N^{\beta}(1+\oslash)\right)
$$

7.8 Proposition For standard sequences $\left(N^{\beta}\right)_{\beta \in \mathbb{N}},\left(\lambda^{\beta}\right)_{\beta \in \mathbb{N}},\left(\delta^{\beta}\right)_{\beta \in \mathbb{N}},\left(\left[0 \ldots T^{\beta}\right]\right)_{\beta \in \mathbb{N}}$ and for standard $h$, the Hypotheses 7.3 and 7.7 are equivalent.

Proof Proposition 7.8 is a consequence of transfer (using that $\left[0 \ldots T^{\beta}\right] \subset\left[0, T^{\beta}\right] \Leftrightarrow$ $\left.T^{\beta} \neq 0\right)$. 
7.9 Theorem Suppose that the Hypotheses 7.3 and 7.4 are fulfilled. Then

$$
\forall f \in \mathcal{C}_{b}(\mathbb{R}) \quad \forall \psi^{*} \in \mathbb{S}^{*}\left(\mathbb{R}^{\mathcal{K}(h)}\right) \quad \lim _{\beta \rightarrow \infty} \mathbb{E}\left[f \circ \psi^{*}\left(\Gamma_{T^{\beta}}^{\beta}\right)\right]=\int_{y \in \mathbb{R}} f(y) \frac{\exp \left(-y^{2} / 2\right)}{\sqrt{2 \pi}} d y .
$$

For any sequence $\left(\nu^{\beta}\right)_{\beta \in \mathbb{N}} \in(0, \infty)^{\mathbb{N}}$ with

$$
\begin{array}{r}
\lim _{\beta \rightarrow \infty} \nu^{\beta}=\infty, \lim _{\beta \rightarrow \infty} \nu^{\beta} / \lambda^{\beta}=0, \nu^{\beta} / \lambda^{\beta} \in\left(0 \ldots T^{\beta}\right) \\
\text { and } T^{\beta}-\nu^{\beta} / \lambda^{\beta} \in\left(\nu^{\beta} / \lambda^{\beta} \ldots T^{\beta}\right)
\end{array}
$$

we obtain

$\forall \varepsilon>0 \exists b \in \mathbb{N} \forall \beta>b \forall k \in \mathcal{K}(h)$

$\mathbb{P}\left(\max _{t \in\left[\nu^{\beta} / \lambda^{\beta} \ldots T^{\beta}\right]} \frac{\left|\Xi_{t, k}^{\beta}-\left(\Phi_{-t}^{\beta} \circ \Gamma_{T^{\beta}}^{\beta}\right)_{k}\right|^{2}}{\left|\left(\Phi_{-t}^{\beta} \circ \Gamma_{T^{\beta}}^{\beta}\right)_{k}\right|^{2}} \geq \frac{4 e^{-\nu^{\beta}}}{\left|\Gamma_{T^{\beta}, k}^{\beta}\right|^{2}} \wedge \Gamma_{T^{\beta}, k}^{\beta} \neq 0\right) \leq e^{-\nu^{\beta}}(1+\varepsilon)$

and $\forall t_{0}^{\beta} \in\left[\nu^{\beta} / \lambda^{\beta} \ldots T^{\beta}-\nu^{\beta} / \lambda^{\beta}\right]$

$$
\mathbb{P}\left(\max _{t \in\left[0 \ldots t_{0}^{\beta}\right]}\left(\Xi_{t, k}^{\beta}\right)^{2} \geq e^{-\lambda^{\beta}\left(T^{\beta}-t_{0}^{\beta}\right)}\right) \leq e^{-\lambda^{\beta}\left(T^{\beta}-t_{0}^{\beta}\right)}(1+\varepsilon) .
$$

Proof The theorem is a statement of ZFC. By an application of transfer we suppose without loss of generality that all objects named in the theorem (including $h$ ) are standard. Thus, by Proposition 7.8, Hypotheses 7.3 implies that Hypothesis 7.7 holds.

The Hypotheses 7.4 and 7.7 imply together with standardness of $h$ that for any $\beta \in \overline{\mathbb{N}}$ the hypotheses of Theorem 6.5 are fulfilled with $N, \lambda, \delta$ and $[0 \ldots T]$ replaced by $N^{\beta}, \lambda^{\beta}, \delta^{\beta}$ and $\left[0 \ldots T^{\beta}\right]$, respectively. Thus also the conclusions of Theorem 6.5 are fulfilled with the respective replacements, i.e.,

$$
\forall \beta \in \overline{\mathbb{N}} \quad \Gamma_{T^{\beta}}^{\beta} \sim_{\oslash} N\left(0, i d_{\mathcal{K}(h)}\right)
$$

and for any sequence $\left(\nu^{\beta}\right)_{\beta \in \mathbb{N}} \in(0, \infty)^{\mathbb{N}}$ such that

$$
\begin{aligned}
& (\forall \beta \in \overline{\mathbb{N}}) \\
& \nu^{\beta} \in \overline{\mathbb{N}}, \quad \nu^{\beta} / \lambda^{\beta} \approx 0, \quad \nu^{\beta} / \lambda^{\beta} \in\left(0 \ldots T^{\beta}\right) \text { and } T^{\beta}-\nu^{\beta} / \lambda^{\beta} \in\left(\nu^{\beta} / \lambda^{\beta} \ldots T^{\beta}\right)
\end{aligned}
$$


we have

$$
\begin{array}{r}
(\forall \beta \in \overline{\mathbb{N}}) \mathbb{P}\left(\max _{t \in\left[\nu^{\beta} / \lambda^{\beta} \ldots T^{\beta}\right]} \frac{\left|\Xi_{t, k}^{\beta}-\left(\Phi_{-t}^{\beta} \circ \Gamma_{T^{\beta}}^{\beta}\right)_{k}\right|^{2}}{\left|\left(\Phi_{-t}^{\beta} \circ \Gamma_{T^{\beta}}^{\beta}\right)_{k}\right|^{2}} \geq \frac{4 e^{-\nu^{\beta}}}{\left|\Gamma_{T^{\beta}, k}^{\beta}\right|^{2}} \wedge \Gamma_{T^{\beta}, k}^{\beta} \neq 0\right) \\
\leq_{\oslash} e^{-\nu^{\beta}}(1+\oslash),
\end{array}
$$

i.e., (97) implies (98). Since (93) implies (97)—by standardness of the involved sequences-and (98) implies (by the permanence principle) (94), the formula (94) has been derived. The proof of (95) is similar to the proof of (94) and thus omitted. It remains to prove (92). Formula (96) is by Remark 4.24 equivalent with

$$
\begin{aligned}
(\forall \beta \in \overline{\mathbb{N}})\left(\forall^{s t} f \in \mathcal{C}_{b}(\mathbb{R})\right) & \left(\forall \psi^{*} \in \mathbb{S}^{*}\left(\mathbb{R}^{\mathcal{K}(h)}\right)\right) \\
& \left(\mathbb{E}\left[f \circ \psi^{*}\left(\Gamma_{T^{\beta}}^{\beta}\right)\right] \approx \int_{y \in \mathbb{R}} f(y) \frac{\exp \left(-y^{2} / 2\right)}{\sqrt{2 \pi}} d y\right)
\end{aligned}
$$

which further implies by Definition 4.2, Remark 4.3 and standardness of the sequence $\left(\Gamma_{T^{\beta}}^{\beta}\right)_{\beta \in \mathbb{N}}$ that:

$$
\begin{aligned}
&\left(\forall^{s t} f \in \mathcal{C}_{b}(\mathbb{R})\right)\left(\forall^{s t} \psi^{*} \in \mathbb{S}^{*}\left(\mathbb{R}^{\mathcal{K}(h)}\right)\right) \\
&\left(\lim _{\beta \rightarrow \infty} \mathbb{E}\left[f \circ \psi^{*}\left(\Gamma_{T^{\beta}}^{\beta}\right)\right]=\int_{y \in \mathbb{R}} f(y) \frac{\exp \left(-y^{2} / 2\right)}{\sqrt{2 \pi}} d y\right)
\end{aligned}
$$

By transfer and the fact that the sequence $\left(\Gamma_{T^{\beta}}^{\beta}\right)_{\beta \in \mathbb{N}}$ is standard the formulas (100) and (92) are equivalent and consequently (92) has been proved.

7.10 Remark The meaning of the phrase 'converges in distribution' used in Corollary 7.11 is introduced in Definition B.2.

7.11 Corollary Under the hypotheses of Theorem 7.9 the sequence $\left(\Gamma_{T^{\beta}}^{\beta}\right)_{\beta \in \mathbb{N}}$ of random variables $\Gamma_{T^{\beta}}^{\beta}$ converges in distribution to an $N(0, i d)$ distributed random variable $\Gamma^{\infty}$ on $\mathbb{R}^{\mathcal{K}(h)}$.

Proof Corollary 7.11 follows by application of Proposition B.4 to (92). It can also be derived by application of B.6 and transfer to the sequence $\left(\Gamma_{T^{\beta}}\right)_{\beta \in \mathbb{N}}$, since for $\beta \in \overline{\mathbb{N}}$ we have by (99) that $\Gamma_{T^{\beta}} \sim_{\oslash} N\left(0, i d_{\mathcal{K}(h)}\right)$. 


\section{A An internal central limit theorem}

A.1 Proposition (Compare with [28, Proposition 3.4]) Let $\sigma \in(0, \infty)$ be limited. A random variable $Y: \Omega \rightarrow \mathbb{R}$ is approximately $N\left(0, \sigma^{2}\right)$ distributed if and only if:

$$
\left(\forall^{s t} g \in \mathcal{C}_{b}^{\infty}(\mathbb{R})\right) \quad\left(\mathbb{E}[g \circ Y] \approx \int_{y \in \mathbb{R}} g(y) \frac{\exp \left(-y^{2} /\left(2 \sigma^{2}\right)\right)}{\sqrt{2 \pi} \sigma} d y\right)
$$

Proof Since $\mathcal{C}_{b}^{\infty}(\mathbb{R}) \subseteq \mathcal{C}_{b}(\mathbb{R})$ it is by Definition 4.18 clear that for an approximately $N\left(0, \sigma^{2}\right)$ distributed random variable $Y$ formula (101) holds. To prove the proposition it thus remains to show the converse, i.e., to prove that (101) implies (15). Let $f \in \mathcal{C}_{b}(\mathbb{R})$ be an arbitrary standard function and let $\varepsilon \in(0, \infty)$ be standard but otherwise arbitrarily chosen. Then there exists a standard $n \in \mathbb{N}$ such that

$$
\int_{y \in \mathbb{R} \backslash[-n+1, n-1]} \frac{\exp \left(-y^{2} /\left(2 \sigma^{2}\right)\right)}{\sqrt{2 \pi} \sigma} d y<\frac{\varepsilon}{6\|f\|} .
$$

Let $\chi: \mathbb{R} \rightarrow[0,1]$ be a standard $\mathcal{C}_{b}^{\infty}(\mathbb{R})$-functions with $\chi(y)=0$ for $y \in[-n+1, n-1]$ and $\chi(y)=1$ for $y \in \mathbb{R} \backslash[-n, n]$ with $n \in \underline{\mathbb{N}}$ arbitrary. We obtain from (101) applied with $g=\chi$ and (102) that $\mathbb{E}(\chi \circ Y) \leq_{\oslash} \frac{\varepsilon}{6\|f\|}+\oslash$ and thus further that

$$
\mathbb{P}(Y \notin[-n, n]) \leq_{\oslash} \frac{\varepsilon}{6\|f\|}+\oslash .
$$

Application of the theorem of Stone Weierstrass (see Willard [29, Theorem 44.6] or Segal and Kunze [26, Theorem 5.1]) and transfer shows the existence of a standard function $g \in \mathcal{C}_{b}^{\infty}(\mathbb{R})$ such that

$$
\sup _{y \in[-n, n]}|f(y)-g(y)| \leq \frac{\varepsilon}{12 n} \text { and }\|g\|_{\infty} \leq\|f\|_{\infty} .
$$

From (101), (102), (103) and (104) we obtain that

$$
\left|\mathbb{E}[f \circ Y]-\int_{y \in \mathbb{R}} f(y) \frac{\exp \left(-y^{2} /\left(2 \sigma^{2}\right)\right)}{\sqrt{2 \pi} \sigma} d y\right| \leq_{\oslash} \varepsilon+\oslash .
$$

Since $f \in \mathcal{C}_{b}(\mathbb{R})$ and $\varepsilon>0$ are standard but otherwise arbitrarily chosen we conclude (15) from (105).

A.2 Definition Given a function $v: \mathbb{R} \rightarrow \mathbb{R}$ we write $v^{\prime \prime}(x)$ to denote the second order derivative of $v$ at $x$. In doing so we implicitly assume that this derivative exists. In the case that $v$ depends additionally on further parameters we use $\frac{\partial^{2}}{\partial x^{2}} v$ to denote the second order derivative of $v$ with respect to $x$. Given $u:[0, S] \times \mathbb{R} \rightarrow \mathbb{R}$ we write $u_{s}\left(\right.$.) to denote the function $u_{s}: \mathbb{R} \rightarrow \mathbb{R}$ given by $u_{s}(x):=u(s, x)$. We further 
write $\dot{u}_{s}($.$) to denote the pointwise derivative of the function s \mapsto u_{S}($.$) , i.e., we let$ $\dot{u}_{s}(x):=\lim _{r \rightarrow 0} \frac{u_{s+r}(x)-u_{s}(x)}{r}$. By displaying $\dot{u}_{s}$ we implicitly assume that the pointwise derivative exists.

A.3 Proposition Let $f \in \mathcal{C}_{b}^{2}(\mathbb{R})$. Suppose that $f^{\prime \prime}$ is uniformly $S$-continuous and limited (which is especially the case for standard $f$ with uniformly continuous second derivative $\left.f^{\prime \prime}\right)$. Let $S \in(0, \infty)$. A solution $u:[0, S] \times \mathbb{R} \rightarrow \mathbb{R}$ of

$$
\dot{u}_{S}(x)+\frac{u_{s}^{\prime \prime}(x)}{2}=0 \text { with } u_{S}(x)=f(x)
$$

exists and is for $s \in[0, S)$ given by

$u_{s}(x)=[f \star \phi](x):=\int f(y) \phi_{s}(x-y) d y$ with $\phi_{s}(z):=\frac{1}{\sqrt{2 \pi(S-s)}} \exp \left(-\frac{z^{2}}{2(S-s)}\right)$.

Further $u_{s}^{\prime \prime}=f^{\prime \prime} \star \phi_{s}$ and the functions $(s, x) \mapsto u_{s}^{\prime \prime}(x)$ and $(s, x) \mapsto \dot{u}_{s}(x)$ are uniformly $S$-continuous and limited on $[0, S] \times \mathbb{R}$.

Proof That (107) is a solution of (106) is well known and easily calculated. That $u_{s}^{\prime \prime}=\left(f \star \phi_{s}\right)^{\prime \prime}=f^{\prime \prime} \star \phi_{s}$ is an easily calculated special case of a well known result in the theory of generalized functions (see Rudin [22, Theorem 6.30]). Since $f^{\prime \prime}$ is uniformly S-continuous and limited the assertion concerning $(s, x) \mapsto u_{s}^{\prime \prime}(x)$ is obtained from $u_{s}^{\prime \prime}=f^{\prime \prime} \star \phi_{s}$ and the special form of the convolution kernels $\phi_{s}$. (Note that the

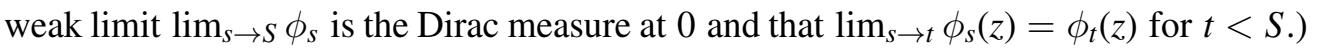
The assertion concerning $(s, x) \mapsto \dot{u}_{s}(x)$ then follows from (106).

A.4 Lemma Let $f \in \mathcal{C}_{b}^{2}(\mathbb{R})$ be standard with uniformly continuous second derivative. Let $S \in \mathbb{R}$ be limited. Let $\Theta$ be an abelian group and let $\psi^{*}: \Theta \rightarrow \mathbb{R}$ be a group homomorphism, i.e., let $\psi^{*}(\theta+\vartheta)=\psi^{*}(\theta)+\psi^{*}(\vartheta)$. Let $\left(W_{s}: \Omega \rightarrow \Theta\right)_{s \in[0 \ldots S]}$ be a finite Markov chain. Let $\mathbb{W}:=\left\{(s, \theta) \mid \mathbb{P}\left(W_{s}=\theta\right)>0\right\}$. For $(s, \theta) \in \mathbb{W}$ let

$$
\delta W_{s, \theta}:=\left.\left(W_{s+\delta s}-\theta\right)\right|_{W_{s}=\theta}
$$

and suppose that for $(s, \theta) \in \mathbb{W}$

(108) $\psi^{*}\left(\delta W_{s, \theta}\right) \approx 0, \quad \mathbb{E}\left[\psi^{*}\left(\delta W_{s, \theta}\right)\right]=0$ and $\mathbb{E}\left[\left(\psi^{*}\right)^{2}\left(\delta W_{s, \theta}\right)\right]=_{\oslash}(1+\oslash) \delta s$.

Let $\hat{u}^{f \circ \psi^{*}}: \mathbb{W} \rightarrow \mathbb{R}$ be recursively defined by

$$
\hat{u}^{f \circ \psi^{*}}(S, \theta):=\left[f \circ \psi^{*}\right](\theta) \text { and } \hat{u}^{f \circ \psi^{*}}(s, \theta):=\mathbb{E}\left[\hat{u}^{f \circ \psi^{*}}\left(s+\delta s, \theta+\delta W_{s, \theta}\right)\right]
$$

If $u$ is a solution of (106) then

$$
\forall s \in[0 \ldots S] \max _{\{\theta \mid(s, \theta) \in \mathbb{W}\}}\left|\hat{u}_{s}^{f \circ \psi^{*}}(\theta)-\left[u_{s} \circ \psi^{*}\right](\theta)\right| \approx 0 .
$$


Proof To simplify notation we let $\hat{u}:=\hat{u}^{f \circ \psi^{*}}$. Since $x \mapsto u_{s+\delta s}^{\prime \prime}(x)$ is by Proposition A.3 uniformly S-continuous and $\psi^{*}\left(\delta W_{s, \theta}\right) \approx \oslash$ a second order expansion of $u\left(s+\delta s, \psi^{*}(\theta)+\psi^{*}\left(\delta W_{s, \theta}\right)\right)$ with respect to $\psi^{*}\left(\delta W_{s, \theta}\right)$ gives

$$
\begin{aligned}
u(s+ & \left.\delta s, \psi^{*}(\theta)+\psi^{*}\left(\delta W_{s, \theta}\right)\right)-u\left(s+\delta s, \psi^{*}(\theta)\right) \\
& ={ }_{\oslash} \psi^{*}\left(\delta W_{s, \theta}\right) \cdot u^{\prime}\left(s+\delta s, \psi^{*}(\theta)\right)+\left(\psi^{*}\left(\delta W_{s, \theta}\right)\right)^{2} \cdot\left(\frac{u^{\prime \prime}\left(s+\delta s, \psi^{*}(\theta)\right)}{2}+\oslash\right)
\end{aligned}
$$

Using the linearity of $\mathbb{E}[$.$] we calculate$

$$
\begin{aligned}
& \mathbb{E}\left[u\left(s+\delta s, \psi^{*}(\theta)+\psi^{*}\left(\delta W_{s, \theta}\right)\right)\right]-u\left(s+\delta s, \psi^{*}(\theta)\right) \\
& \quad \stackrel{(a)}{=} \mathbb{E}\left[\left(\psi^{*}\left(\delta W_{s, \theta}\right)\right)^{2}\right] \cdot\left(\frac{u^{\prime \prime}\left(s+\delta s, \psi^{*}(\theta)\right)}{2}+\oslash\right) \\
& \stackrel{(b)}{=}-(1+\oslash) \delta s\left[\dot{u}\left(s+\delta s, \psi^{*}(\theta)\right)+\oslash\right] \stackrel{(c)}{=}-\delta s\left[\dot{u}\left(s+\delta s, \psi^{*}(\theta)\right)+\oslash\right]
\end{aligned}
$$

with (a) a consequence of (111) and (108), (b) a consequence of (106) and (108), and (c) a consequence of the fact that $s \mapsto \dot{u}_{s+\delta s}\left(\psi^{*}(\theta)\right)$ is by Proposition A.3 limited.

By the fact that $s \mapsto \dot{u}_{s+\delta s}\left(\psi^{*}(\theta)\right)$ is by Proposition A.3 uniformly S-continuous a first order expansion of $u\left(s+\delta s, \psi^{*}(\theta)\right)$ with respect to $-\delta s$ gives

$$
u\left(s+\delta s, \psi^{*}(\theta)\right)-u\left(s, \psi^{*}(\theta)\right)={ }_{\varnothing} \delta s\left[\dot{u}\left(s+\delta s, \psi^{*}(\theta)\right)+\oslash\right] .
$$

By adding (112) and (113) we obtain that

$$
\left|\mathbb{E}\left[u\left(s+\delta s, \psi^{*}(\theta)+\psi^{*}\left(\delta W_{s, \theta}\right)\right)\right]-u\left(s, \psi^{*}(\theta)\right)\right| \leq_{\oslash} \delta s \oslash .
$$

We calculate using that $\psi^{*}$ is a group homomorphism

$$
\begin{aligned}
\left|\hat{u}_{s}(\theta)-\left[u_{s} \circ \psi^{*}\right](\theta)\right| \stackrel{(a)}{=}\left|\mathbb{E}\left[\hat{u}_{s+\delta s}\left(\theta+\delta W_{s, \theta}\right)\right]-\left[u_{s} \circ \psi^{*}\right](\theta)\right| \\
\leq\left|\mathbb{E}\left[\hat{u}_{s+\delta s}\left(\theta+\delta W_{s, \theta}\right)\right]-\mathbb{E}\left[\left[u_{s+\delta s} \circ \psi^{*}\right]\left(\theta+\delta W_{s, \theta}\right)\right]\right| \\
\quad+\mid \mathbb{E}\left[u_{s+\delta s}\left(\psi^{*}(\theta)+\psi^{*}\left(\delta W_{s, \theta}\right)\right]-u_{s}\left(\psi^{*}(\theta)\right) \mid\right. \\
\stackrel{(b)}{\leq} \max _{\oslash}\left|\hat{u}_{s+\delta s}(\vartheta)-\left[u_{s+\delta s} \circ \psi^{*}\right](\vartheta)\right|+\delta s \oslash
\end{aligned}
$$

with (a) a consequence of (109) and (b) a consequence of (114). From (115) we obtain that

$$
\max _{\{\theta \mid(s, \theta) \in \mathbb{W}\}}\left|\hat{u}_{s}(\theta)-\left[u_{s} \circ \psi^{*}\right](\theta)\right| \leq_{\oslash} \max _{\{\theta \mid(s+\delta s, \theta) \in \mathbb{W}\}}\left|\hat{u}_{s+\delta s}(\theta)-\left[u_{s+\delta s} \circ \psi^{*}\right](\theta)\right|+\delta s \oslash .
$$

Since by (109) and (106)

$$
\hat{u}_{S}(.)=\left[f \circ \psi^{*}\right](.)=\left[u_{S} \circ \psi^{*}\right](.)
$$


we conclude from (116) using backward induction and limitedness of $S$ that (110) holds.

A.5 Theorem Let $\Theta$ be an abelian group and let $\left(W_{s}: \Omega \rightarrow \Theta\right)_{s \in[0 \ldots S]}$ be a finite Markov chain. Let $\mathbb{W}:=\left\{(s, \theta) \mid \mathbb{P}\left(W_{s}=\theta\right)>0\right\}$ and for $(s, \theta) \in \mathbb{W}$ let

$$
\delta W_{s, \theta}:=\left.\left(W_{s+\delta s}-\theta\right)\right|_{W_{s}=\theta} .
$$

Suppose that we are given a function $\hat{f}: \Theta \rightarrow \mathbb{R}$ and that the function $\hat{u} \hat{f}^{\prime}: \mathbb{W} \rightarrow \mathbb{R}$ is recursively defined by

$$
\hat{u}^{\hat{f}}(S, \theta)=\hat{f}(\theta) \text { and } \hat{u}^{\hat{f}}(s, \theta)=\mathbb{E}\left[\hat{u}^{\hat{f}}\left(s+\delta s, \theta+\delta W_{s, \theta}\right)\right]
$$

Then for $(s, \theta) \in \mathbb{W}$ we have that

$$
\mathbb{E}\left[\hat{f} \circ W_{S} \mid W_{s}=\theta\right]=\hat{u}_{s}^{\hat{f}}(\theta) .
$$

Suppose that $\psi^{*}: \Theta \rightarrow \mathbb{R}$ denotes a group homomorphism such that $\delta W_{s, \theta}$ fulfills (108) and suppose that $W_{0}=0$. Then $\psi^{*} \circ W_{S}$ is approximately $N(0, S)$ distributed and

$$
\mathbb{E}\left[\left(\psi^{*}\right)^{2} \circ W_{S}\right]={ }_{\oslash}(1+\oslash) S .
$$

A.6 Remark Note that an approximately $N(0,1)$ distributed random variable does not necessarily posses a second moment. Thus it is necessary to prove (119) separately.

Proof of Theorem A.5 Equation (118) is proved by backward induction on [0..S]. The induction hypothesis is correct for $t=S$ since by the definition of conditional expectation and by (117)

$$
\mathbb{E}\left[\hat{f} \circ W_{S} \mid W_{S}=\theta\right]=\hat{f}(\theta)=\hat{u}_{S}^{\hat{f}}(\theta) .
$$

Suppose next that the induction hypothesis holds for $t=s+\delta s$, i.e., that for $(s+\delta s, \vartheta) \in$ $\mathbb{W}$

$$
\mathbb{E}\left[\hat{f} \circ W_{S} \mid W_{s+\delta s}=\vartheta\right]=\hat{u}_{s+\delta s}^{\hat{f}}(\vartheta)
$$

Then for $(s, \theta) \in \mathbb{W}$

$$
\begin{gathered}
\mathbb{E}\left[\hat{f} \circ W_{S} \mid W_{s}=\theta\right]=\sum_{\{\vartheta \mid(s+\delta s, \vartheta) \in \mathbb{W}\}} \mathbb{P}\left(W_{s+\delta s}=\vartheta \mid W_{s}=\theta\right) \cdot \mathbb{E}\left[\hat{f} \circ W_{S} \mid W_{s+\delta s}=\vartheta\right] \\
=\sum_{\{\vartheta \mid(s+\delta s, \vartheta) \in \mathbb{W}\}} \mathbb{P}\left(\theta+\delta W_{s, \theta}=\vartheta\right) \cdot \hat{u}_{s+\delta s}^{\hat{f}}(\vartheta)=\mathbb{E}\left[\hat{u}_{s+\delta s}^{\hat{f}}\left(\theta+\delta W_{s, \theta}\right)\right] \stackrel{(117)}{=} \hat{u}_{s}^{\hat{f}}(\theta)
\end{gathered}
$$

i.e., (118) holds for $t=s$. Thus (118) has been proved by backward induction. 
We prove next that $\psi^{*} \circ W_{S}$ is approximately $N(0, S)$ distributed. To do this let $f \in \mathcal{C}_{b}^{\infty}$ be an arbitrary standard function and let $\hat{f}=f \circ \psi^{*}$. Then the hypotheses of Lemma A.4 hold and thus also its conclusion (110) is fulfilled. Consequently we calculate

$$
\begin{aligned}
\mathbb{E}\left[f \circ \psi^{*} \circ W_{S}\right] \stackrel{(a)}{=} \mathbb{E}\left[\hat{f} \circ W_{S} \mid W_{0}=0\right] & \stackrel{(b)}{=} \hat{u}_{0}^{\hat{f}}(0)=\hat{u}_{0}^{f \circ \psi^{*}}(0) \\
& \stackrel{(c)}{\approx} u_{0}(0) \stackrel{(d)}{=} \int f(y) \frac{1}{\sqrt{2 \pi S}} \exp \left(-\frac{y^{2}}{2 S}\right) d y
\end{aligned}
$$

where (a) follows from the hypothesis $W_{0}=0$, (b) is a consequence of (118), (c) a consequence of (110), and (d) follows from (107). From (120) and Proposition A.1 we obtain that $\psi^{*} \circ W_{S}$ is approximately $N(0, S)$ distributed.

Finally to show (119) we calculate (using that $\psi^{*}$ is a group homomorphism)

$$
\begin{aligned}
\mathbb{E}\left[\left(\psi^{*}\right)^{2}\right. & \left.\circ W_{s+\delta s}\right]=\sum_{\{\theta \mid(s, \theta) \in \mathbb{W}\}} \mathbb{E}\left[\left(\psi^{*}\left(\delta W_{s, \theta}+\theta\right)\right)^{2} \mid W_{s}=\theta\right] \cdot \mathbb{P}\left(W_{s}=\theta\right) \\
& =\sum_{\{\theta \mid(s, \theta) \in \mathbb{W}\}} \mathbb{E}\left[\left(\psi^{*}\left(\delta W_{s, \theta}\right)\right)^{2}+2 \psi^{*}\left(\delta W_{s, \theta}\right) \cdot \psi^{*}(\theta)+\left(\psi^{*}(\theta)\right)^{2}\right] \cdot \mathbb{P}\left(W_{s}=\theta\right) \\
& \stackrel{(a)}{=}(1+\oslash) \delta s+\mathbb{E}\left[\left(\psi^{*}\right)^{2} \circ W_{s}\right]
\end{aligned}
$$

with (a) a consequence of (108) and the linearity of $\mathbb{E}[$.$] . From (121) we obtain by$ induction along the near interval $[0 \ldots S)$ and since $W_{0}=0$ that (119) holds.

A.7 Theorem Let $[0 \ldots T]$ be a near interval and let $t_{0} \in[0 \ldots T]$. Let $\Theta$ be an abelian group and let $\psi^{*}: \Theta \rightarrow \mathbb{R}$ be a group homomorphism. Let $\left(\Gamma_{t}\right)_{t \in[0 \ldots T]}$ be a Markov process with values in $\Theta$ such that $\Gamma_{0}=0$. Let $\mathbb{G}:=\left\{(t, \theta) \mid \mathbb{P}\left(\Gamma_{t}=\theta\right)>0\right\}$ and for $(t, \theta) \in \mathbb{G}$ let

$$
\delta \Gamma_{t, \theta}:=\left.\left(\Gamma_{t+\delta t}-\theta\right)\right|_{\Gamma_{t}=\theta} .
$$

Suppose that $\psi^{*} \circ \delta \Gamma_{t, \theta} \approx 0, \mathbb{E}\left[\psi^{*} \circ \delta \Gamma_{t, \theta}\right]=0, \mathbb{E}\left[\left(\psi^{*}\right)^{2} \circ \delta \Gamma_{t, \theta}\right]={ }_{\diamond}(1+\oslash) \sigma_{t}^{2}$ with $\sigma_{t}^{2}$ infinitesimal and such that $\sum_{t \in[0 \ldots T)} \sigma_{t}^{2}$ is limited. Then

$$
\begin{gathered}
\mathbb{E}\left[\left(\psi^{*}\right)^{2} \circ \Gamma_{t_{0}}\right]=\sum_{\theta \in\left[0 \ldots t_{0}\right)} \sigma_{t}^{2}(1+\oslash), \quad \psi^{*} \circ \Gamma_{t_{0}} \sim_{\oslash} N\left(0, \sum_{t \in\left[0 \ldots t_{0}\right)} \sigma_{t}^{2}\right) \\
\mathbb{E}\left[\left(\psi^{*}\right)^{2} \circ\left(\Gamma_{T}-\Gamma_{t_{0}}\right)\right]=\sum_{\oslash} \sum_{t \in\left[t_{0} \ldots T\right)} \sigma_{t}^{2}(1+\oslash) \text { and } \\
\psi^{*} \circ\left(\Gamma_{T}-\Gamma_{t_{0}}\right) \sim_{\oslash} N\left(0, \sum_{t \in\left[t_{0} \ldots T\right)} \sigma_{t}^{2}\right) .
\end{gathered}
$$


Proof To prove (122) we define a function

$$
\iota:\left[0 \ldots t_{0}\right] \rightarrow[0, \infty) \text { by } \iota(t):=\sum_{u \in[0 \ldots t)} \sigma_{u}^{2}
$$

let $[0 \ldots S]:=\left\{\iota(t) \mid t \in\left[0 \ldots t_{0}\right]\right\}$ and note that $S=\iota\left(t_{0}\right)=\sum_{t \in\left[0 \ldots t_{0}\right)} \sigma_{t}^{2}$. Let $\left(W_{s}\right)_{s \in[0 \ldots S]}$ be the Markov process defined by $W_{\iota(t)}=\Gamma_{t}$. Then $\left(W_{s}\right)_{s \in[0 \ldots S]}$ fulfills the hypotheses of Theorem A.5. Thus application of Theorem A.5 shows that

$$
\psi^{*} \circ \Gamma_{t_{0}}=\psi^{*} \circ W_{S} \sim_{\oslash} N(0, S)=N\left(0, \sum_{t \in\left[0 \ldots t_{0}\right)} \sigma_{t}^{2}\right)
$$

and

$$
\mathbb{E}\left[\left(\psi^{*}\right)^{2} \circ \Gamma_{t_{0}}\right]=\mathbb{E}\left[\left(\psi^{*}\right)^{2} \circ W_{S}\right]={ }_{\oslash}(1+\oslash) S=\sum_{\oslash \in\left[0 \ldots t_{0}\right)} \sigma_{t}^{2}(1+\oslash)
$$

i.e., (122) has been proved. To prove (123) it suffices by ${ }^{10}$

$$
\psi^{*} \circ\left(\Gamma_{T}-\Gamma_{t_{0}}\right)=\left.\sum_{\left\{\gamma \mid\left(t_{0}, \gamma\right) \in \mathbb{G}\right\}}^{\circ} \psi^{*} \circ\left(\Gamma_{T}-\gamma\right)\right|_{\Gamma_{t_{0}}=\gamma}
$$

to prove that

$$
\begin{array}{r}
\mathbb{P}\left(\Gamma_{t_{0}}=\gamma\right)>\left.0 \Rightarrow \psi^{*} \circ\left(\Gamma_{T}-\gamma\right)\right|_{\Gamma_{t_{0}}=\gamma} \sim_{\oslash} N\left(0, \sum_{t \in\left[t_{0} \ldots T\right)} \sigma_{t}^{2}\right) \\
\text { and } \mathbb{E}\left[\left.\left(\psi^{*}\right)^{2} \circ\left(\Gamma_{T}-\gamma\right)\right|_{\Gamma_{t_{0}}=\gamma}\right]=\sum_{t \in\left[t_{0} \ldots T\right)} \sigma_{t}^{2}(1+\oslash) .
\end{array}
$$

The proof of (126) is analogous to the proof of (122).

\section{B Connection to standard mathematics}

B.1 Remark In this appendix we make use of general measure theoretic probability theory. Probabilities, expectations etc. are defined and used in the sense of standard measure theoretic probability theory (see for example Dudley [9]) that coincides in the case of finite probability spaces with our definitions. For a nonstandard characterization of weak convergence relating the subject to Loeb measure theory see Anderson and Rashid [2].

\footnotetext{
${ }^{10}$ The symbol $\sum^{\circ}$ in $(125)$ denotes the formation of a disjoint union; note that we identify functions with their graphs.
} 
B.2 Definition Given a topological space $\mathcal{X}$. We say that a sequence $\left(X_{n}\right)_{n \in \mathbb{N}}$ of random variables $X_{n}: \Omega_{n} \rightarrow \mathcal{X}$ converges in distribution to $X_{\infty}$, if there exists a random variable $X_{\infty}: \Omega_{\infty} \rightarrow \mathcal{X}$ such that

$$
\left(\forall f \in \mathcal{C}_{b}(\mathcal{X})\right) \lim _{n \rightarrow \infty} \mathbb{E}\left[f \circ X_{n}\right]=\mathbb{E}\left[f \circ X_{\infty}\right]
$$

B.3 Proposition Suppose that we are given a standard sequence $\left(X_{n}\right)_{n \in \mathbb{N}}$ of random variables $X_{n}: \Omega_{n} \rightarrow \mathcal{X}$ and a standard random variable $X_{\infty}: \Omega_{\infty} \rightarrow \mathcal{X}$. The sequence $\left(X_{n}\right)_{n \in \mathbb{N}}$ converges in distribution to $X_{\infty}$ if and only if

$$
\left(\forall^{s t} f \in \mathcal{C}_{b}(\mathcal{X})\right)(\forall n \in \overline{\mathbb{N}}) \mathbb{E}\left[f \circ X_{n}\right] \approx \mathbb{E}\left[f \circ X_{\infty}\right] .
$$

Proof By transfer and standardness of $\left(X_{n}\right)_{n \in \mathbb{N}}$ and $X_{\infty}$ we obtain that (127) is equivalent with

$$
\left(\forall^{s t} f \in \mathcal{C}_{b}(\mathcal{X})\right) \lim _{n \rightarrow \infty} \mathbb{E}\left[f \circ X_{n}\right]=\mathbb{E}\left[f \circ X_{\infty}\right]
$$

For standard $f \in \mathcal{C}_{b}(\mathcal{X})$ the sequence $\left(\mathbb{E}\left[f \circ X_{n}\right]\right)_{n \in \mathbb{N}}$ is also standard. Thus by application of Definition 4.2 and Remark 4.3 we obtain that (129) and (128) are equivalent. All together we have proved the equivalence of (127) and (128) and conclude the assertion of the proposition from Definition (B.2).

B.4 Proposition Let $\mathcal{J}$ be a finite set. A sequence $\left(X_{n}\right)_{n \in \mathbb{N}}$ of random variables $X_{n}: \Omega \rightarrow \mathbb{R}^{\mathcal{J}}$ converges in distribution to an $N(0, i d)$ distributed random variable $X_{\infty}$ if and only if:

$$
\left(\forall f \in \mathcal{C}_{b}(\mathbb{R})\right)\left(\forall \psi^{*} \in \mathbb{S}^{*}\left(\mathbb{R}^{\mathcal{J}}\right)\right)\left(\lim _{n \rightarrow \infty} \mathbb{E}\left[f \circ \psi^{*}\left(X_{n}\right)\right]=\int_{y \in \mathbb{R}} f(y) \frac{\exp \left(-y^{2} / 2\right)}{\sqrt{2 \pi}} d y\right)
$$

Proof The proposition is a consequence of the Cramér-Wold device (see Pollard [20, Chapter 8, Sections 6 and 7] or Van der Vaart [27, Section 2, before Example 2.18]).

B.5 Remark Definition 4.22 is justified by the following implication of Proposition B.4.

B.6 Proposition Let $\mathcal{J}$ be a standard finite set and let $\left(X_{n}\right)_{n \in \mathbb{N}}$ be a standard sequence of random variables $X_{n}: \Omega \rightarrow \mathbb{R}^{\mathcal{J}}$ such that

$$
(\forall n \in \overline{\mathbb{N}}) X_{n} \sim_{\oslash} N\left(0, i d_{\mathcal{J}}\right)
$$

Then $\left(X_{n}\right)_{n \in \mathbb{N}}$ converges in distribution to an $N(0, i d)$ distributed random variable $X_{\infty}$. 
Proof Formula (131) implies by Remark 4.24 that:

$$
\left(\forall^{s t} f \in \mathcal{C}_{b}(\mathbb{R})\right)\left(\forall^{s t} \psi^{*} \in \mathbb{S}^{*}\left(\mathbb{R}^{\mathcal{J}}\right)\right)\left(\mathbb{E}\left[\left[f \circ \psi^{*}\right]\left(X_{n}\right)\right] \approx \int_{y \in \mathbb{R}} f(y) \frac{\exp \left(-y^{2} / 2\right)}{\sqrt{2 \pi}} d y\right)
$$

For standard $f$ and $\psi^{*}$ we obtain by standardness of $\left(X_{n}\right)_{n \in \mathbb{N}}$ that the sequence $\left(\mathbb{E}\left(\left[f \circ \psi^{*}\right]\left(X_{n}\right)\right)\right)_{n \in \mathbb{N}}$ is standard. Thus by Definition 4.2 and Remark 4.3 we obtain that (132) is equivalent with:

$$
\begin{aligned}
\left(\forall^{s t} f \in \mathcal{C}_{b}(\mathbb{R})\right)\left(\forall^{s t} \psi^{*}\right. & \left.\in \mathbb{S}^{*}\left(\mathbb{R}^{\mathcal{J}}\right)\right) \\
& \left(\lim _{n \rightarrow \infty} \mathbb{E}\left[\left[f \circ \psi^{*}\right]\left(X_{n}\right)\right]=\int_{y \in \mathbb{R}} f(y) \frac{\exp \left(-y^{2} / 2\right)}{\sqrt{2 \pi}} d y\right)
\end{aligned}
$$

By standardness of $\left(X_{n}\right)_{n \in \mathbb{N}}$ an application of transfer to (133) shows that (130) holds. Application of Proposition B.4 completes the proof.

\section{Miscellaneous Results}

C.1 Proposition Suppose that $\kappa \in \mathbb{R}$, that $t \in(0, \infty)$ is limited and that $\delta \in(0, \infty)$ is infinitesimal. Suppose further that $\kappa^{2} \delta \approx 0$. Then

$$
(1+\kappa \delta)^{2 t / \delta}={ }_{\oslash} e^{2 \kappa t}(1+\oslash) \text {. }
$$

Proof Since $\frac{e^{2 \kappa t}}{(1+\kappa \delta)^{2 t / \delta}}=\left(\frac{e^{\kappa \delta}}{1+\kappa \delta}\right)^{2 t / \delta}$ the following calculation proves the result:

$$
\begin{aligned}
1 & \stackrel{(a)}{\leq}\left(\frac{e^{\kappa \delta}}{1+\kappa \delta}\right)^{2 t / \delta}=\left(1+\frac{e^{\kappa \delta}-(1+\kappa \delta)}{(1+\kappa \delta)}\right)^{2 t / \delta} \\
& \stackrel{(b)}{=}\left(1+\kappa^{2} \delta^{2} /(2+\oslash)\right)^{2 t / \delta} \stackrel{(c)}{\leq}\left(e^{\kappa^{2} \delta^{2} /(2+\oslash)}\right)^{2 t / \delta}={ }_{\oslash} e^{2 t \kappa^{2} \delta /(2+\oslash)} \stackrel{(d)}{=} \stackrel{1}{\oslash}_{1+\oslash}
\end{aligned}
$$

Note that $(a)$ and $(c)$ follow since the exponential function is convex and thus its graph lies strictly above its tangent at $0,(b)$ follows from Taylor series expansion of the exponential function around 0 since $\kappa \delta \approx 0$, and finally $(d)$ follows from $t \kappa^{2} \delta \approx 0$, the S-continuity of the exponential function around 0 and that $(1+\oslash)={ }_{\oslash} \frac{1}{1+\oslash}$.

C.2 Proposition Let $\widetilde{\eta}:[x, x+h] \rightarrow \mathbb{R}$ be differentiable and denote by $\widetilde{\eta}^{\prime}$ its derivative. Then

$$
\left(\frac{\widetilde{\eta}(x+h)-\widetilde{\eta}(x)}{h}\right)^{2} \leq \frac{1}{h} \int_{x}^{x+h}\left(\widetilde{\eta}^{\prime}\right)^{2}(y) d y
$$


Proof

$$
\frac{\widetilde{\eta}(x+h)-\widetilde{\eta}(x)}{h}=\int_{0}^{1} \widetilde{\eta}^{\prime}(x+s \cdot h) d s .
$$

Thus by Jensen's inequality

$$
\left(\frac{\widetilde{\eta}(x+h)-\widetilde{\eta}(x)}{h}\right)^{2} \leq \int_{0}^{1}\left(\widetilde{\eta}^{\prime}\right)^{2}(x+s \cdot h) d s=\frac{1}{h} \int_{x}^{x+h}\left(\widetilde{\eta}^{\prime}\right)^{2}(y) d y .
$$

C.3 Proposition (Doob inequality) Let $\left(Z_{t}\right)_{t \in\left[t_{0} \ldots T\right]}$ be a martingale with $Z_{t_{0}}=0$. Then

$$
\mathbb{P}\left(\max _{t \in\left[t_{0} \ldots T\right]} Z_{t}^{2} \geq \varepsilon>0\right) \leq \frac{1}{\varepsilon} \mathbb{E}\left[Z_{T}^{2}\right]
$$

Proof The Doob inequality can bee found in many textbooks on probability theory. See for example Nelson [19, Theorem 11.4].

Acknowledgement: I would like to thank the referees for their remarks, that helped to improve the readability of the article.

\section{References}

[1] S Albeverio, JE Fenstad, R Høegh-Krohn, T Lindstrøm, Nonstandard Methods in Stochastic Analysis and Mathematical Physics, Academic Press, New York, 1986.

[2] R M Anderson, S Rashid, A nonstandard characterization of weak convergence, Proc. Am. Math. Soc. 69(1978), 327-332; doi:10.2307/2042621.

[3] A G Angel, M R Evans, E Levine, D Mukamel, Criticality and condensation in a non-conserving zero-range process, J. Stat. Mech. P08017(2007); doi:10.1088/17425468/2007/08/P08017.

[4] R. Bhattacharya, M. Majumdar, Random Dynamical Systems: Theory and Applications, Cambridge University Press, 2007.

[5] I van den Berg, Nonstandard Asymptotic Analysis, Lecture Notes in Mathematics 1249, Springer-Verlag, 1987.

[6] C Boldrighini, A De Masi, A Pellegrinotti, Nonequilibrium fluctuations in particle systems modelling reaction-diffusion equations, Stochastic Processes Appl. 42, No.1(1992), 1-30; doi:10.1016/0304-4149(92)90023-J.

[7] M.-F. Chen, From Markov Chains to Non-equilibrium Particle Systems (2nd ed.) World Scientific, River Edge, NJ, 2004. 
[8] F Diener, M Diener, Tutorial, in Nonstandard Analysis in Practice (eds. F Diener, M Diener), Universitext, Springer-Verlag, Berlin, 1995.

[9] R M Dudley, Real Analysis and Probability, Cambridge Studies in Advanced Mathematics 74, Cambridge University Press, 2002.

[10] M R Evans, T Hanney, Nonequilibrium statistical mechanics of the zero-range process and related models, J. Phys. A, Math. Gen. 38, No. 19(2005), R195-R240; doi:10.1088/0305-4470/38/19/R01.

[11] D Griffeath, Additive and Cancellative Interacting Particle Systems, Lecture Notes in Mathematics 724, Springer-Verlag, Berlin-Heidelberg-New York, 1979.

[12] L L Helms, P A Loeb, Applications of nonstandard analysis to spin models, J. Math. Anal. Appl. 69(1979), 341-352; doi:10.1016/0022-247X(79)90147-1.

[13] V Kanovei, M Reeken, Nonstandard Analysis, Axiomatically, Springer Monographs in Mathematics, Springer, Berlin, 2004.

[14] F Koudjeti, I van den Berg, Neutrices, external numbers, and external calculus, in Nonstandard Analysis in Practice (eds. F Diener, M Diener), Universitext, SpringerVerlag, Berlin, 1995.

[15] T M Liggett, Interacting Particle Systems, Reprint of 1985 edition with a new postface, Classics in Mathematics, Springer, New York, 2005.

[16] T Lindstrøm, An invitation to nonstandard analysis, in Nonstandard Analysis and its Applications (ed. N J Cutland), Lond. Math. Soc. Stud. Texts 10, Cambridge University Press, 1988, 1-105.

[17] Luong, Bao, Fourier Analysis on Finite Abelian Groups, Applied and Numerical Harmonic Analysis, Birkhäuser, Boston, MA, 2009.

[18] E Nelson, Internal set theory: A new approach to nonstandard analysis, Bull. Am. Math. Soc. 83(1977), 1165-1198 ; doi:10.1090/S0002-9904-1977-14398-X.

[19] E Nelson, Radically Elementary Probability Theory, Annals of Mathematics Studies, No.117, Princeton University Press, Princeton, New Jersey, 1987.

[20] D Pollard, A User's Guide to Measure Theoretic Probability, Cambridge Series in Statistical and Probabilistic Mathematics 8, Cambridge University Press, 2002.

[21] A Robinson, Non-standard Analysis, reprinted by Princeton Univ. Press, Princeton, NJ, 1996.

[22] W Rudin, Functional Analysis, 2nd ed., International Series in Pure and Applied Mathematics, McGraw-Hill, New York, 1991.

[23] F Sagues, J M Sancho, J Garcia-Ojalvo, Spatiotemporal order out of noise, Rev. Mod. Phys., 79(3)(2007), 829-882; doi:10.1103/RevModPhys.79.829.

[24] K Stroyan, J Bayod, Foundations of Infinitesimal Stochastic Analysis, Studies in Logic and the Foundations of Mathematics, Vol. 119, North-Holland, Amsterdam-New York-Oxford, 1986. 
[25] A Terras, Fourier Analysis on Finite Groups and Applications, London Mathematical Society Student Texts 43, Cambridge University Press, 1999.

[26] I Segal, R Kunze, Integrals and Operators, (2nd ed.), Springer, Berlin, 1978.

[27] A W van der Vaart, Asymptotic Statistics, Cambridge Series in Statistical and Probabilistic Mathematics 3, Cambridge Univ. Press, 1998.

[28] H Weisshaupt, Diffusion processes via parabolic equations: an infinitesimal approach to Lindeberg's limit theorem, J. Log. Anal. 1:2(2009), 1-29; doi:10.4115/jla.2009.1.2.

[29] S Willard, General Topology, Addison-Wesley, Mass., 1970.

ZBSA University of Freiburg, Habsburgerstrasse 49, 79104 Freiburg, Germany

heinz.weisshaupt@zbsa.uni-freiburg.de,

heinz.weisshaupt@univie.ac.at

Received: 31 March $2010 \quad$ Revised: 17 May 2011 